Supporting Information

\title{
Synergistic Acid-Promoted Synthesis of Highly Substituted Butenolides via the Annulation of Keto Acids and Tertiary Alcohols
}

Wenbin Mao, ${ }^{\dagger}$ and Chen $\mathrm{Zhu}^{*,+, \dagger}$

${ }^{\dagger}$ Key Laboratory of Organic Synthesis of Jiangsu Province, College of Chemistry, Chemical Engineering and Materials Science, Soochow University, 199 Ren-Ai Road, Suzhou, Jiangsu 215123,

People's Republic of China

Key Laboratory of Synthetic Chemistry of Natural Substances, Shanghai Institute of Organic Chemistry, Chinese Academy of Sciences, 345 Lingling Road, Shanghai 200032, People's Republic of China

Email: $\underline{\text { chzhu@ @uda.edu.cn }}$

\section{Table of Contents}

$\begin{array}{ll}\text { 1. General experimental details } & \text { S2 }\end{array}$

$\begin{array}{ll}\text { 2. General procedure for the synthesis of butenolides } & \text { S2 }\end{array}$

$\begin{array}{ll}3 . & \text { Characterization of products }\end{array}$

$\begin{array}{ll}\text { 4. KIE experiment } & \text { S9 }\end{array}$

5. ${ }^{1} \mathrm{H},{ }^{13} \mathrm{C}$, and ${ }^{19} \mathrm{~F}$ NMR spectra $\quad \mathrm{S} 10$ 


\section{General experimental details}

Commercially available reagents were used without further purification. Infrared (FT-IR) spectra were recorded on a BRUKER VERTEX 70, vmax in $\mathrm{cm}^{-1}$. ${ }^{1} \mathrm{H}-\mathrm{NMR}$ spectra were recorded on a BRUKER AVANCE III HD (400 MHz) spectrometer. Chemical shifts are reported in ppm from tetramethylsilane with the solvent resonance as internal standard (CDCl3: $\delta$ 7.26). Data are reported as follows: chemical shift, multiplicity $(\mathrm{s}=$ singlet, $\mathrm{d}=$ doublet, $\mathrm{t}=$ triplet, $\mathrm{q}=$ quadruplet, $\mathrm{br}=\mathrm{broad}, \mathrm{m}=$ multiplet), coupling constants $(\mathrm{Hz})$ and integration. ${ }^{13} \mathrm{C}-\mathrm{NMR}$ spectra were recorded on a BRUKER AVANCE III HD (100 MHz) spectrometer with complete proton decoupling. Chemical shifts are reported in ppm from tetramethylsilane with the solvent resonance as the internal standard (CDCl3: $\delta$ 77.16). ${ }^{19}$ F-NMR spectra were recorded on a BRUKER AVANCE III HD (376 MHz) spectrometer. Mass spectra were measured with an Agelent Technologies 6120 Quadrupole LC/MS. High resolution mass spectrometry (HRMS) were measured with a GCT Premier ${ }^{\mathrm{TM}}$ and BRUKER micrOTF-Q III. Melting points were measured using INESA WRR and values are uncorrected.

Keto acids were prepared by the oxidation of acetophenone derivatives according to the reported procedure. ${ }^{1}$

\section{General procedure for the synthesis of butenolides}

Keto acid 1 ( $0.3 \mathrm{mmol}, 1$ equiv) and $p \mathrm{TSA}^{\mathrm{H}} \mathrm{H}_{2} \mathrm{O}(0.75 \mathrm{mmol}, 2.5$ equiv) were loaded in a test tube, which was added $2 \mathrm{~mL}$ of $p$-xylene as solvent. Then, tertiary alcohol 2 (0.45 mmol, 1.5 equiv) and $\mathrm{BF}_{3} \mathrm{Et}_{2} \mathrm{O}\left(0.06 \mathrm{mmol}, 0.2\right.$ equiv) were added to the solution. The reaction mixture was stirred at $110{ }^{\circ} \mathrm{C}$ until the starting material had been consumed as determined by TLC. Then the reaction was cooled to room temperature. After removal of the solvent under vacuum, the residue was subjected to column chromatography on silica gel (ethyl acetate/ petroleum ether) to give the butenolide $\mathbf{3}$.

\section{Characterization of products}

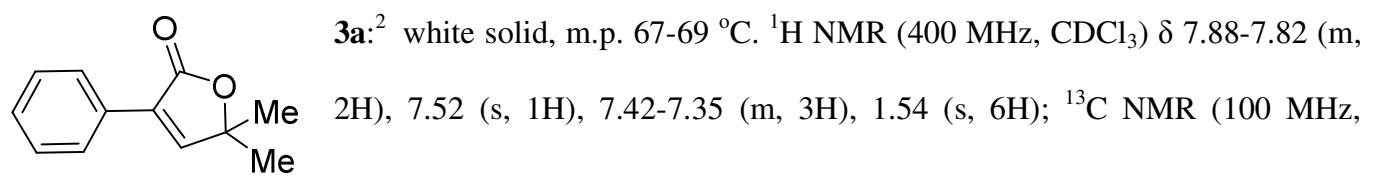

\footnotetext{
${ }^{1}$ (a) Beebe, X.; Nilius, A. M.; Merta, P. J.; Soni, N. B.; Bui, M. H.; Wagner, R.; Beutel, B. A. Bioorg. Med. Chem. Lett. 2003, 13, 3133-3136. (b) Zhuang, J.; Wang, C.; Xie, F.; Zhang, W. Tetrahedron 2009, 65, 9797-9800. (c) Johannes, E.; Horbert, R.; Schlosser, J.; Schmidt, D.; Peifer, C. Tetrahedron Lett. 2013, 54, 4067-4072.

2 Arcadi, A.; Bernocchi, E.; Burini, A.; Cacchi, S.; Marinelli, F.; Pietroni, B. Tetrahedron 1988, 44, 481-490.
} 
$\left.\mathrm{CDCl}_{3}\right) \delta 171.2,153.1,129.9,129.6,129.2,128.6,127.1,83.4,25.7 . \mathrm{ESI}[\mathrm{M}+\mathrm{H}]^{+} 189.1$.

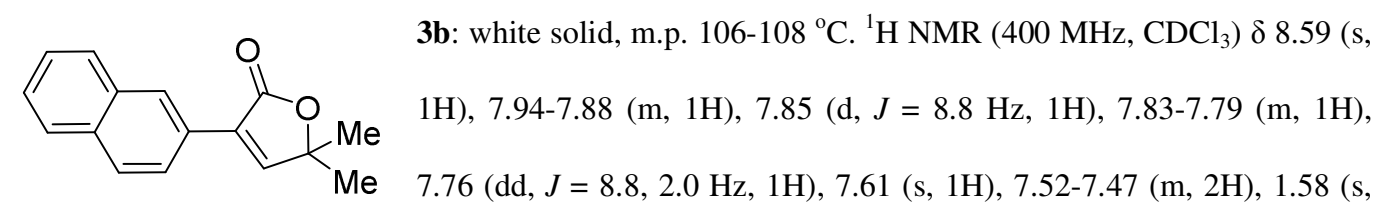
$6 \mathrm{H}) ;{ }^{13} \mathrm{C} \mathrm{NMR}\left(100 \mathrm{MHz}, \mathrm{CDCl}_{3}\right) \delta 170.7,152.5,132.9,132.7,129.3,128.3,127.8,127.1,126.5$, 126.4, 126.3, 126.0, 123.7, 83.0, 25.3. FT-IR: $v\left(\mathrm{~cm}^{-1}\right) 3058,2976,1731,1575,1462,1361$. HRMS [CI] calcd for $\mathrm{C}_{16} \mathrm{H}_{15} \mathrm{O}_{2}[\mathrm{M}+\mathrm{H}]^{+}$239.1072, found 239.1075; ESI $[\mathrm{M}+\mathrm{H}]^{+} 239.1$.

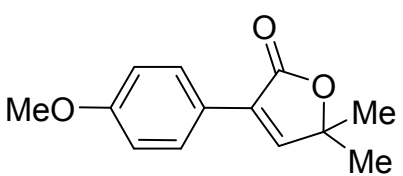

3c: yellow solid, m.p. $57-59{ }^{\circ} \mathrm{C} .{ }^{1} \mathrm{H}$ NMR $\left(400 \mathrm{MHz}, \mathrm{CDCl}_{3}\right) \delta$ 7.84-7.80 (m, 2H), $7.38(\mathrm{~s}, 1 \mathrm{H}), 6.94-6.90(\mathrm{~m}, 2 \mathrm{H}), 3.83(\mathrm{~s}, 3 \mathrm{H}), 1.54$ $(\mathrm{s}, 6 \mathrm{H}) ;{ }^{13} \mathrm{C} \mathrm{NMR}\left(100 \mathrm{MHz}, \mathrm{CDCl}_{3}\right) \delta 171.0,159.8,150.3,128.9$, 128.0, 121.7, 113.5, 82.8, 54.8, 25.4. FT-IR: $v\left(\mathrm{~cm}^{-1}\right)$ 3087, 2982, 1732, 1573, 1461, 1363. HRMS [CI] calcd for $\mathrm{C}_{13} \mathrm{H}_{15} \mathrm{O}_{3}[\mathrm{M}+\mathrm{H}]^{+}$219.1021, found 219.1029; ESI [M+H $]^{+} 219.1$.

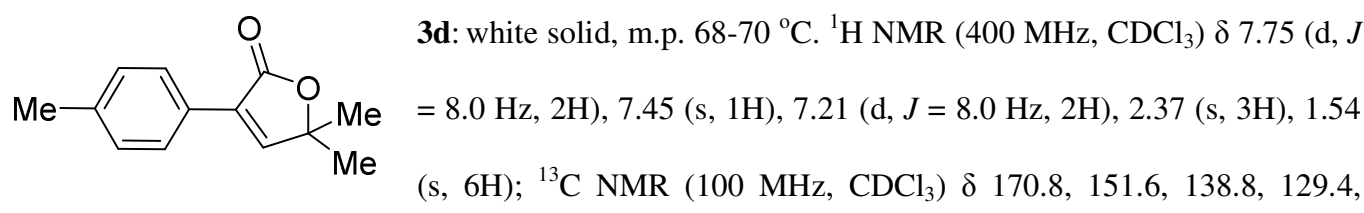
128.8, 126.5, 126.3, 82.9, 25.3, 20.9. FT-IR: $v\left(\mathrm{~cm}^{-1}\right) 3079,2985,1734,1513,1444,1364$. HRMS [CI] calcd for $\mathrm{C}_{13} \mathrm{H}_{15} \mathrm{O}_{2}[\mathrm{M}+\mathrm{H}]^{+}$203.1072, found 203.1069; ESI $[\mathrm{M}+\mathrm{H}]^{+}$203.1.

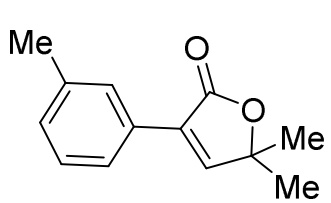

3e: yellow oil. ${ }^{1} \mathrm{H}$ NMR (400 MHz, $\left.\mathrm{CDCl}_{3}\right) \delta 7.67(\mathrm{~s}, 1 \mathrm{H}), 7.63(\mathrm{~d}, J=7.6$ $\mathrm{Hz}, 1 \mathrm{H}), 7.49$ (s, 1H), $7.29(\mathrm{dd}, J=7.6,7.6 \mathrm{~Hz}, 1 \mathrm{H}), 7.19$ (d, $J=7.6 \mathrm{~Hz}$, $1 \mathrm{H}), 2.38(\mathrm{~s}, 3 \mathrm{H}), 1.54(\mathrm{~s}, 6 \mathrm{H}) ;{ }^{13} \mathrm{C} \mathrm{NMR}\left(100 \mathrm{MHz}, \mathrm{CDCl}_{3}\right) \delta 171.1,152.9$, 138.3, 130.1, 130.0, 129.5, 128.5, 127.7, 124.2, 83.4, 25.8, 21.5. FT-IR: $v\left(\mathrm{~cm}^{-1}\right) 3063,2979,1747$, 1586, 1461, 1365. HRMS [CI] calcd for $\mathrm{C}_{13} \mathrm{H}_{15} \mathrm{O}_{2}[\mathrm{M}+\mathrm{H}]^{+}$203.1072, found 203.1076; ESI [M+H] ${ }^{+}$ 203.1. 


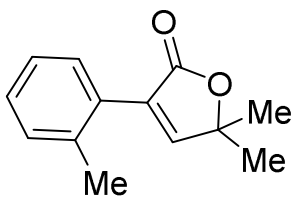

3f: white solid, m.p. $90-92{ }^{\circ} \mathrm{C} .{ }^{1} \mathrm{H}$ NMR $\left(400 \mathrm{MHz}, \mathrm{CDCl}_{3}\right) \delta 7.36(\mathrm{~d}, J=7.6$ $\mathrm{Hz}, 1 \mathrm{H}), 7.29(\mathrm{~s}, 1 \mathrm{H}), 7.29-7.19(\mathrm{~m}, 3 \mathrm{H}), 2.33(\mathrm{~s}, 3 \mathrm{H}), 1.58(\mathrm{~s}, 6 \mathrm{H}) ;{ }^{13} \mathrm{C}$ NMR

$\left(100 \mathrm{MHz}, \mathrm{CDCl}_{3}\right) \delta 171.0,156.1,136.0,131.2,130.0,129.1,129.0,128.4$ 125.4, 83.5, 25.4, 19.9. FT-IR: $v\left(\mathrm{~cm}^{-1}\right)$ 3029, 2976, 1728, 1597, 1462, 1361. HRMS [CI] calcd for $\mathrm{C}_{13} \mathrm{H}_{15} \mathrm{O}_{2}[\mathrm{M}+\mathrm{H}]^{+}$203.1072, found 203.1079; ESI [M+H $]^{+} 203.1$.

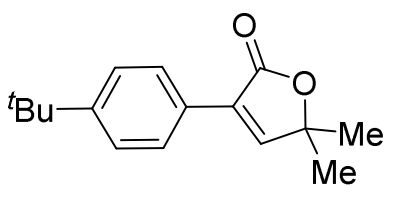

3g: white solid, m.p. $91-93{ }^{\circ} \mathrm{C} .{ }^{1} \mathrm{H}$ NMR $\left(400 \mathrm{MHz}, \mathrm{CDCl}_{3}\right) \delta 7.81-7.76$ $(\mathrm{m}, 2 \mathrm{H}), 7.47(\mathrm{~s}, 1 \mathrm{H}), 7.45-7.40(\mathrm{~m}, 2 \mathrm{H}), 1.54(\mathrm{~s}, 6 \mathrm{H}), 1.33(\mathrm{~s}, 9 \mathrm{H}) ;{ }^{13} \mathrm{C}$ NMR $\left(100 \mathrm{MHz}, \mathrm{CDCl}_{3}\right) \delta 170.8,152.0,151.8,129.4,126.4,126.3$, 125.1, 82.9, 34.2, 30.7, 25.3. FT-IR: $v\left(\mathrm{~cm}^{-1}\right)$ 3087, 2955, 1735, 1507, 1461, 1363. HRMS [ESI] calcd for $\mathrm{C}_{16} \mathrm{H}_{21} \mathrm{O}_{2}[\mathrm{M}+\mathrm{H}]^{+}$245.1542, found 245.1538; ESI $[\mathrm{M}+\mathrm{H}]^{+} 245.1$.

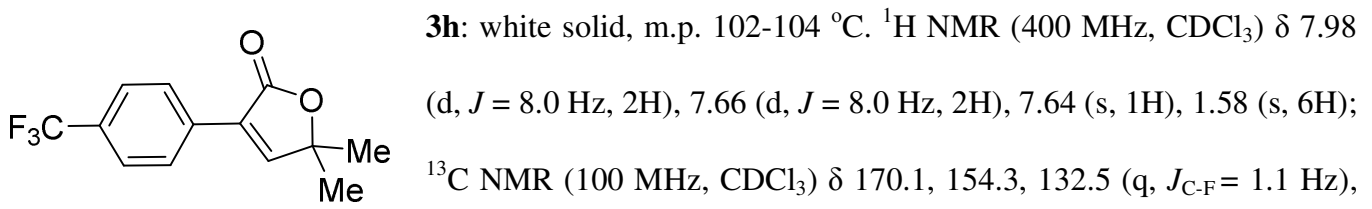
$130.5\left(\mathrm{q}, J_{\mathrm{C}-\mathrm{F}}=32.4 \mathrm{~Hz}\right), 128.5,127.0,125.1\left(\mathrm{q}, J_{\mathrm{C}-\mathrm{F}}=3.7 \mathrm{~Hz}\right), 123.4\left(\mathrm{q}, J_{\mathrm{C}-\mathrm{F}}=270 \mathrm{~Hz}\right), 83.3,25.1 ;{ }^{19} \mathrm{~F}$ NMR (376 MHz, $\left.\mathrm{CDCl}_{3}\right) \delta-62.8$ (s). FT-IR: $v\left(\mathrm{~cm}^{-1}\right)$ 3066, 2988, 1739, 1575, 1463, 1369. HRMS [CI] calcd for $\mathrm{C}_{13} \mathrm{H}_{12} \mathrm{O}_{2} \mathrm{~F}_{3}[\mathrm{M}+\mathrm{H}]^{+}$257.0789, found 257.0784; ESI [M+H] $]^{+} 257.1$.

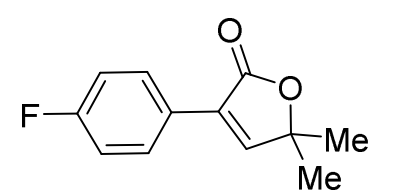

3i: white solid, m.p. 82-84 ${ }^{\circ} \mathrm{C} .{ }^{1} \mathrm{H}$ NMR $\left(400 \mathrm{MHz}, \mathrm{CDCl}_{3}\right) \delta 7.89-7.82$ $(\mathrm{m}, 2 \mathrm{H}), 7.47(\mathrm{~s}, 1 \mathrm{H}), 7.13-7.05(\mathrm{~m}, 2 \mathrm{H}), 1.55(\mathrm{~s}, 6 \mathrm{H}) ;{ }^{13} \mathrm{C}$ NMR $(100$

$\left.\mathrm{MHz}, \mathrm{CDCl}_{3}\right) \delta 170.5,162.7\left(\mathrm{~d}, J_{\mathrm{C}-\mathrm{F}}=248 \mathrm{~Hz}\right), 152.0\left(\mathrm{~d}, J_{\mathrm{C}-\mathrm{F}}=1.6 \mathrm{~Hz}\right)$ $128.6\left(\mathrm{~d}, J_{\mathrm{C}-\mathrm{F}}=8.2 \mathrm{~Hz}\right), 128.5,125.2\left(\mathrm{~d}, J_{\mathrm{C}-\mathrm{F}}=3.4 \mathrm{~Hz}\right), 115.2\left(\mathrm{~d}, J_{\mathrm{C}-\mathrm{F}}=21.5 \mathrm{~Hz}\right), 83.0,25.3 ;{ }^{19} \mathrm{~F} \mathrm{NMR}$ $\left(376 \mathrm{MHz}, \mathrm{CDCl}_{3}\right) \delta-111.3$ (s). FT-IR: $v\left(\mathrm{~cm}^{-1}\right)$ 3087, 2981, 1733, 1507, 1448, 1364. HRMS [ESI] calcd for $\mathrm{C}_{12} \mathrm{H}_{12} \mathrm{O}_{2} \mathrm{~F}[\mathrm{M}+\mathrm{H}]^{+}$207.0821, found 207.0819; ESI [M+H] $]^{+} 207.1$.

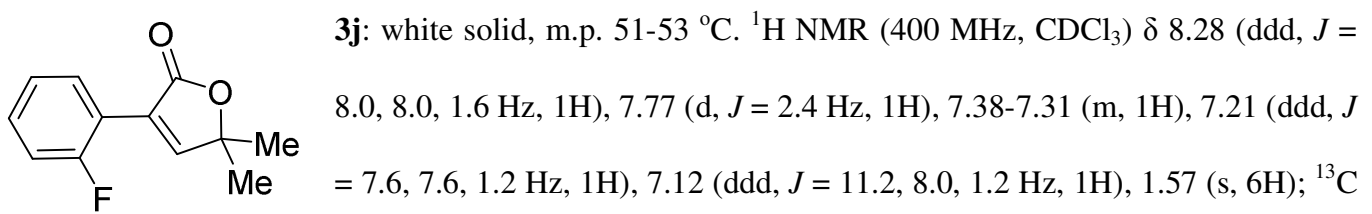
$\operatorname{NMR}\left(100 \mathrm{MHz}, \mathrm{CDCl}_{3}\right) \delta 170.6,160.4\left(\mathrm{~d}, J_{C-F}=150 \mathrm{~Hz}\right), 157.1\left(\mathrm{~d}, J_{C-F}=11.6 \mathrm{~Hz}\right), 130.0\left(\mathrm{~d}, J_{C-F}\right.$ 
$=8.8 \mathrm{~Hz}), 129.1\left(\mathrm{~d}, J_{C-F}=2.3 \mathrm{~Hz}\right), 123.8\left(\mathrm{~d}, J_{C-F}=3.6 \mathrm{~Hz}\right), 122.9\left(\mathrm{~d}, J_{C-F}=1.9 \mathrm{~Hz}\right), 117.2\left(\mathrm{~d}, J_{C-F}\right.$

$=11.5 \mathrm{~Hz}), 115.1\left(\mathrm{~d}, J_{C-F}=22.1 \mathrm{~Hz}\right), 83.3\left(\mathrm{~d}, J_{C-F}=1.6 \mathrm{~Hz}\right), 25.1 ;{ }^{19} \mathrm{~F} \mathrm{NMR}\left(376 \mathrm{MHz}, \mathrm{CDCl}_{3}\right) \delta$ -112.8 (s). FT-IR: $v\left(\mathrm{~cm}^{-1}\right)$ 3083, 2989, 1741, 1571, 1448, 1363. HRMS [CI] calcd for $\mathrm{C}_{12} \mathrm{H}_{12} \mathrm{O}_{2} \mathrm{~F}$ $[\mathrm{M}+\mathrm{H}]^{+}$207.0821, found 207.0823; ESI $[\mathrm{M}+\mathrm{H}]^{+}$207.1.

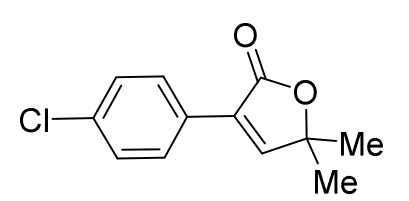

3k: white solid, m.p. 89-91 ${ }^{\circ} \mathrm{C} .{ }^{1} \mathrm{H}$ NMR $\left(400 \mathrm{MHz}, \mathrm{CDCl}_{3}\right) \delta$ 7.82-7.79 $(\mathrm{m}, 2 \mathrm{H}), 7.52(\mathrm{~s}, 1 \mathrm{H}), 7.39-7.36(\mathrm{~m}, 2 \mathrm{H}), 1.56(\mathrm{~s}, 6 \mathrm{H}) ;{ }^{13} \mathrm{C}$ NMR $(100$ $\left.\mathrm{MHz}, \mathrm{CDCl}_{3}\right) \delta 170.4,152.7,134.8,128.5,128.4,127.9,127.5,83.1$, 25.2. FT-IR: $v\left(\mathrm{~cm}^{-1}\right) 3075,2977,1731,1594,1468,1365$. HRMS [CI] calcd for $\mathrm{C}_{12} \mathrm{H}_{12} \mathrm{O}_{2} \mathrm{Cl}[\mathrm{M}+\mathrm{H}]^{+}$ 223.0526, found 223.0527; ESI [M+H] $]^{+} 223.0$.

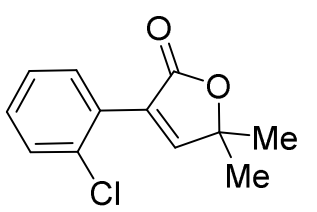

3l: white solid, m.p. $110-112{ }^{\circ} \mathrm{C} .{ }^{1} \mathrm{H}$ NMR $\left(400 \mathrm{MHz}, \mathrm{CDCl}_{3}\right) \delta 7.70(\mathrm{~s}, 1 \mathrm{H})$, 7.68-7.65 (m, 1H), 7.46-7.41 (m, 1H), 7.35-7.26 (m, 2H), $1.59(\mathrm{~s}, 6 \mathrm{H}) ;{ }^{13} \mathrm{C}$ NMR $\left(100 \mathrm{MHz}, \mathrm{CDCl}_{3}\right) \delta 170.6,158.0,132.7,130.4,129.6,129.5,127.9$, 127.5, 126.3, 83.6, 25.1. FT-IR: $v\left(\mathrm{~cm}^{-1}\right)$ 3053, 2980, 1742, 1586, 1467, 1365. HRMS [ESI] calcd for $\mathrm{C}_{12} \mathrm{H}_{11} \mathrm{O}_{2} \mathrm{ClNa}[\mathrm{M}+\mathrm{Na}]^{+} 245.0345$, found 245.0347; ESI [M+H] $]^{+} 223.1$.

3m: white solid, m.p. 85-87 ${ }^{\circ} \mathrm{C} .{ }^{1} \mathrm{H}$ NMR $\left(400 \mathrm{MHz}, \mathrm{CDCl}_{3}\right) \delta 7.74(\mathrm{~d}, J$ 123.5, 83.7, 25.7. FT-IR: $v\left(\mathrm{~cm}^{-1}\right)$ 3083, 2979, 1745, 1593, 1425, 1358. HRMS [CI] calcd for $\mathrm{C}_{12} \mathrm{H}_{12} \mathrm{O}_{2} \mathrm{Br}[\mathrm{M}+\mathrm{H}]^{+}$267.0021, found 267.0018; ESI [M+H] $]^{+}$267.0.

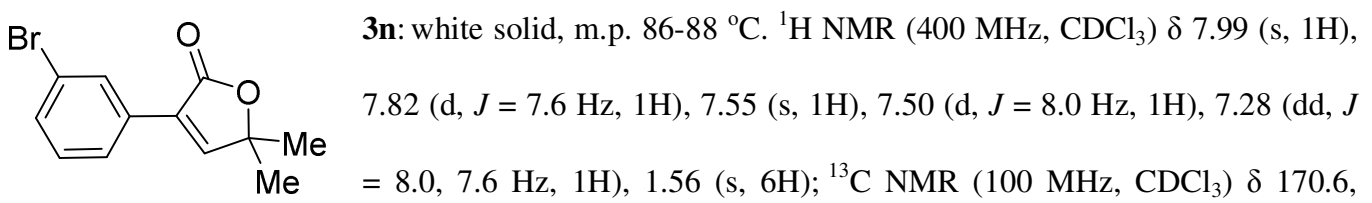
154.0, 132.2, 131.6, 130.2, 130.0, 128.8, 125.7, 122.7, 83.6 25.7. FT-IR: $v\left(\mathrm{~cm}^{-1}\right) 3073,2974,1732$, 1587, 1444, 1364. HRMS [CI] calcd for $\mathrm{C}_{12} \mathrm{H}_{12} \mathrm{O}_{2} \mathrm{Br}[\mathrm{M}+\mathrm{H}]^{+}$267.0021, found 267.0017; ESI [M+H] 267.0. 


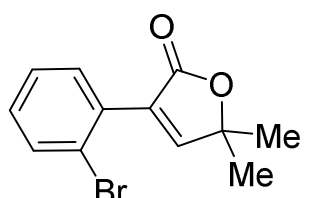

3o: white solid, m.p. $118-120{ }^{\circ} \mathrm{C} .{ }^{1} \mathrm{H}$ NMR $\left(400 \mathrm{MHz}, \mathrm{CDCl}_{3}\right) \delta 7.65$ (dd, $J=$ 7.6, $1.2 \mathrm{~Hz}, 2 \mathrm{H}), 7.64(\mathrm{~s}, 1 \mathrm{H}), 7.53(\mathrm{dd}, J=8.0,1.6 \mathrm{~Hz}, 1 \mathrm{H}), 7.36(\mathrm{ddd}, J=$ 8.0, 7.6, $1.2 \mathrm{~Hz}, 1 \mathrm{H}), 7.22(\mathrm{ddd}, J=8.0,7.6,1.6 \mathrm{~Hz}, 1 \mathrm{H}), 1.60(\mathrm{~s}, 6 \mathrm{H}) ;{ }^{13} \mathrm{C}$ NMR $\left(100 \mathrm{MHz}, \mathrm{CDCl}_{3}\right) \delta 170.4,158.0,132.9,130.5,130.1,129.7,129.6,126.9,122.6,83.7,25.1$. FT-IR: $v\left(\mathrm{~cm}^{-1}\right) 3052,2985,1739,1464,1426,1361$. HRMS [CI] calcd for $\mathrm{C}_{12} \mathrm{H}_{12} \mathrm{O}_{2} \mathrm{Br}[\mathrm{M}+\mathrm{H}]^{+}$ 267.0021, found 267.0022; ESI [M+H] $]^{+} 267.0$.

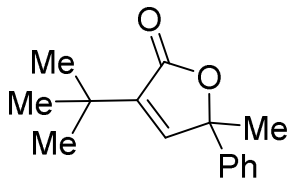

3p: white solid, m.p. 87-89 ${ }^{\circ} \mathrm{C} .{ }^{1} \mathrm{H}$ NMR $\left(400 \mathrm{MHz}, \mathrm{CDCl}_{3}\right) \delta$ 7.39-7.37 (m, 2H), 7.37-7.35 (m, 2H), 7.35-7.29 (m, 1H), $7.14(\mathrm{~s}, 1 \mathrm{H}), 1.77(\mathrm{~s}, 3 \mathrm{H}), 1.26(\mathrm{~s}$, $9 \mathrm{H}) ;{ }^{13} \mathrm{C} \mathrm{NMR}\left(100 \mathrm{MHz}, \mathrm{CDCl}_{3}\right) \delta 170.9,150.1,139.9,139.8,128.3,127.6$, 124.3, 84.7, 30.9, 27.6, 26.4. FT-IR: $v\left(\mathrm{~cm}^{-1}\right)$ 3098, 2960, 1742, 1601, 1467, 1363. HRMS [ESI] calcd for $\mathrm{C}_{15} \mathrm{H}_{18} \mathrm{O}_{2} \mathrm{Na}[\mathrm{M}+\mathrm{Na}]^{+}$253.1204, found 253.1207; ESI [M+H] $]^{+} 231.1$.

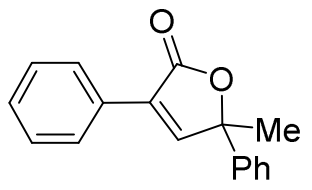

3r: white solid, m.p. $73-75{ }^{\circ} \mathrm{C} .{ }^{1} \mathrm{H}$ NMR $\left(400 \mathrm{MHz}, \mathrm{CDCl}_{3}\right) \delta$ 7.88-7.84 (m, 2H), $7.74(\mathrm{~s}, 1 \mathrm{H}), 7.46-7.42(\mathrm{~m}, 2 \mathrm{H}), 7.41-7.35(\mathrm{~m}, 5 \mathrm{H}), 7.34-7.31(\mathrm{~m}, 1 \mathrm{H})$, $1.88(\mathrm{~s}, 3 \mathrm{H}) ;{ }^{13} \mathrm{C}$ NMR $\left(100 \mathrm{MHz}, \mathrm{CDCl}_{3}\right) \delta 171.1,152.1,139.8,129.4,129.3$, 128.9, 128.7, 128.3, 127.2, 124.9, 85.9, 26.7. FT-IR: $v\left(\mathrm{~cm}^{-1}\right)$ 3068, 2986, 1737, 1601, 1448, 1371. HRMS [CI] calcd for $\mathrm{C}_{17} \mathrm{H}_{15} \mathrm{O}_{2}[\mathrm{M}+\mathrm{H}]^{+}$251.1072, found 251.1073; ESI [M+H] ${ }^{+} 251.1$.

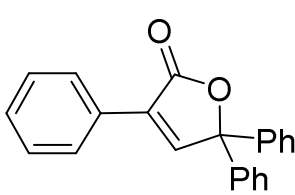

3s: white solid, m.p. $158-160{ }^{\circ} \mathrm{C} .{ }^{1} \mathrm{H}$ NMR $\left(400 \mathrm{MHz}, \mathrm{CDCl}_{3}\right) \delta 8.04(\mathrm{~s}, 1 \mathrm{H})$, 7.92-7.87 (m, 2H), 7.40-7.30 (m, 13H); $\left.{ }^{13} \mathrm{C} \mathrm{NMR} \mathrm{(100} \mathrm{MHz,} \mathrm{CDCl}_{3}\right) \delta 170.4$, $150.0,139.3,129.3,129.1,128.8,128.3,128.2,128.1,126.9,126.3,88.7$. FT-IR: $v\left(\mathrm{~cm}^{-1}\right) 3066,2945,1746,1597,1449,1345$. HRMS [ESI] calcd for $\mathrm{C}_{22} \mathrm{H}_{16} \mathrm{O}_{2} \mathrm{Na}[\mathrm{M}+\mathrm{Na}]^{+}$ 335.1048, found 335.1054; ESI [M+H] $]^{+} 313.1$.

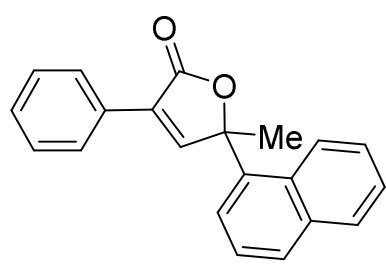

3t: white solid, m.p. $104-106{ }^{\circ} \mathrm{C} .{ }^{1} \mathrm{H}$ NMR $\left(400 \mathrm{MHz}, \mathrm{CDCl}_{3}\right) \delta 8.49(\mathrm{~d}$, $J=8.8 \mathrm{~Hz}, 1 \mathrm{H}), 8.28(\mathrm{~s}, 1 \mathrm{H}), 7.91-7.86(\mathrm{~m}, 3 \mathrm{H}), 7.82(\mathrm{~d}, J=8.0 \mathrm{~Hz}$, 1H), 7.64-7.57 (m, 2H), 7.55-7.49 (m, 1H), 7.44-7.33 (m, 4H), $2.12(\mathrm{~s}$, $3 \mathrm{H}) ;{ }^{13} \mathrm{C} \mathrm{NMR}\left(100 \mathrm{MHz}, \mathrm{CDCl}_{3}\right) \delta 169.9,150.8,135.9,134.2,129.8$, 129.6, 129.3, 129.0, 128.9, 128.2, 126.8, 126.0, 125.3, 124.9, 124.6, 123.1, 86.6, 27.1. FT-IR: $v\left(\mathrm{~cm}^{-1}\right)$ 
3064, 2924, 1741, 1597, 1448, 1349. HRMS [ESI] calcd for $\mathrm{C}_{21} \mathrm{H}_{16} \mathrm{O}_{2} \mathrm{Na}[\mathrm{M}+\mathrm{Na}]^{+} 323.1048$, found 323.1045; ESI [M+H] ${ }^{+} 301.1$.

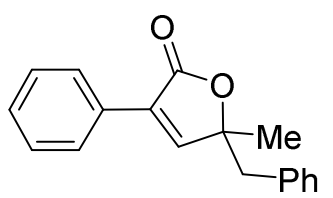

3u: yellow solid, m.p. $71-73{ }^{\circ} \mathrm{C} .{ }^{1} \mathrm{H}$ NMR $\left(400 \mathrm{MHz}, \mathrm{CDCl}_{3}\right) \delta$ 7.74-7.68 (m, 2H), $7.43(\mathrm{~s}, 1 \mathrm{H}), 7.39-7.32(\mathrm{~m}, 3 \mathrm{H}), 7.30-7.25(\mathrm{~m}, 2 \mathrm{H}), 7.25-7.21(\mathrm{~m}, 1 \mathrm{H})$, 7.21-7.17 (m, 2H), $3.10(\mathrm{~s}, 2 \mathrm{H}), 1.53(\mathrm{~s}, 3 \mathrm{H}) ;{ }^{13} \mathrm{C} \mathrm{NMR}\left(100 \mathrm{MHz}, \mathrm{CDCl}_{3}\right) \delta$ 170.4, 151.3, 134.4, 130.7, 129.8, 129.1, 128.7, 128.1, 127.9, 126.7, 126.6, 85.0, 45.1, 23.5. FT-IR: v $\left(\mathrm{cm}^{-1}\right)$ 3085, 2983, 1734, 1601, 1447, 1369. HRMS [CI] calcd for $\mathrm{C}_{18} \mathrm{H}_{17} \mathrm{O}_{2}[\mathrm{M}+\mathrm{H}]^{+} 265.1229$, found 265.1220; ESI $[\mathrm{M}+\mathrm{H}]^{+} 265.1$.

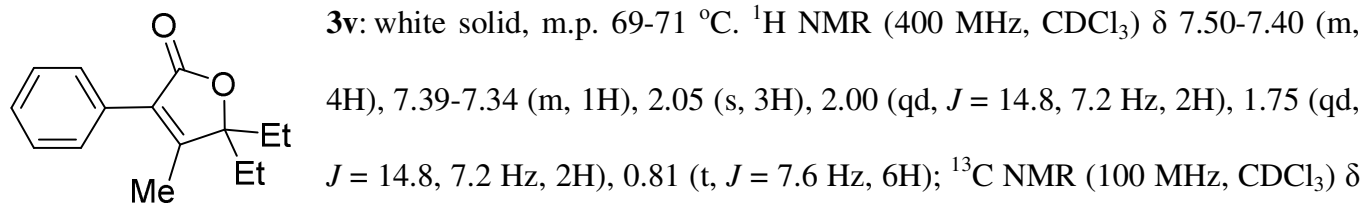
171.8, 161.5, 129.8, 128.5, 128.1, 128.0, 127.8, 89.9, 28.7, 11.8, 6.6. FT-IR: $v\left(\mathrm{~cm}^{-1}\right) 3056,2980,1734$, 1654, 1452, 1353. HRMS [ESI] calcd for $\mathrm{C}_{15} \mathrm{H}_{18} \mathrm{O}_{2} \mathrm{Na}[\mathrm{M}+\mathrm{Na}]^{+}$253.1204, found 253.1201; ESI $[\mathrm{M}+\mathrm{H}]^{+} 231.1$

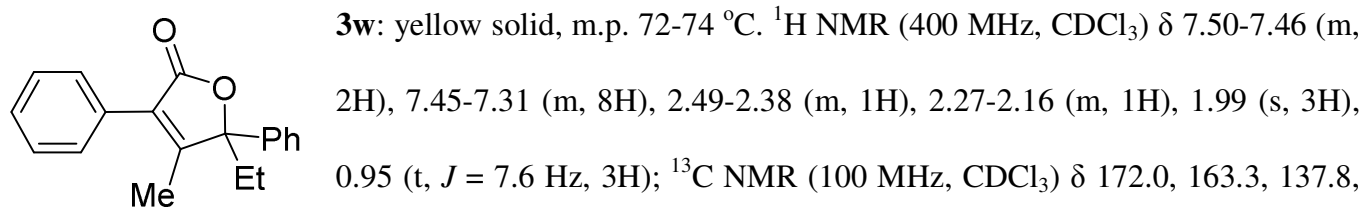
129.6, 128.6, 128.3, 128.0, 127.9, 125.8, 124.9, 89.9, 27.9, 12.1, 7.0. FT-IR: $v\left(\mathrm{~cm}^{-1}\right) 3058,2971,1741$, 1600, 1446, 1332. HRMS [ESI] calcd for $\mathrm{C}_{19} \mathrm{H}_{19} \mathrm{O}_{2}[\mathrm{M}+\mathrm{H}]^{+}$279.1385, found 279.1382; ESI [M+H] $]^{+}$ 279.1.

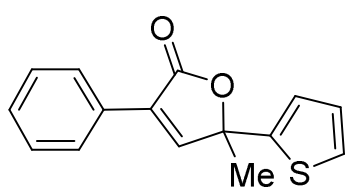

3x: brown oil. ${ }^{1} \mathrm{H}$ NMR (400 MHz, $\left.\mathrm{CDCl}_{3}\right) \delta$ 7.90-7.87 (m, 2H), $7.66(\mathrm{~s}$, 1H), 7.45-7.40 (m, 3H), $7.31(\mathrm{dd}, J=5.2,1.2 \mathrm{~Hz}, 1 \mathrm{H}), 7.08(\mathrm{dd}, J=3.6$, $1.2 \mathrm{~Hz}, 1 \mathrm{H}), 7.00(\mathrm{dd}, J=5.2,3.6 \mathrm{~Hz}, 1 \mathrm{H}), 1.99(\mathrm{~s}, 3 \mathrm{H}) ;{ }^{13} \mathrm{C} \mathrm{NMR}(100$ $\left.\mathrm{MHz}, \mathrm{CDCl}_{3}\right) \delta 170.1,150.4,142.2,129.5,129.1,128.6,128.2,126.8,126.7,125.6,124.5,82.8,26.0$. FT-IR: $v\left(\mathrm{~cm}^{-1}\right)$ 3071, 2980, 1749, 1598, 1447, 1353. HRMS [ESI] calcd for $\mathrm{C}_{15} \mathrm{H}_{12} \mathrm{O}_{2} \mathrm{SNa}[\mathrm{M}+\mathrm{Na}]^{+}$ 279.0456, found 279.0455; ESI [M+H] ${ }^{+} 257.0$ 


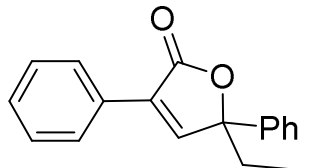

5a: yellow solid, m.p. $82-84{ }^{\circ} \mathrm{C} .{ }^{1} \mathrm{H}$ NMR $\left(400 \mathrm{MHz}, \mathrm{CDCl}_{3}\right) \delta$ 7.88-7.83 (m, 2H), 7.73 (s, 1H), 7.46-7.42 (m, 2H), 7.42-7.35 (m, 5H), 7.34-7.28 (m, 1H), $2.24(\mathrm{qd}, J=14.4,7.6 \mathrm{~Hz}, 1 \mathrm{H}), 2.09(\mathrm{qd}, J=14.4,7.6 \mathrm{~Hz}, 1 \mathrm{H}), 0.94(\mathrm{t}, J=$ $8.0 \mathrm{~Hz}, 3 \mathrm{H}) ;{ }^{13} \mathrm{C} \mathrm{NMR}\left(100 \mathrm{MHz}, \mathrm{CDCl}_{3}\right) \delta 170.7,150.5,138.8,129.7,129.0,128.9,128.3,128.2$, 127.6, 126.7, 124.7, 88.4, 32.7, 7.7. FT-IR: $v\left(\mathrm{~cm}^{-1}\right) 3087,2972,1737,1599,1448,1334$. HRMS [ESI] calcd for $\mathrm{C}_{18} \mathrm{H}_{16} \mathrm{O}_{2} \mathrm{Na}[\mathrm{M}+\mathrm{Na}]^{+}$287.1048, found 287.1043; ESI [M+H] ${ }^{+} 265.1$.

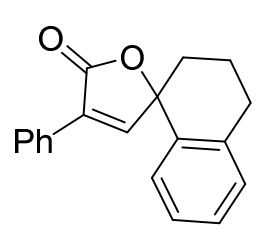

5b: white solid, m.p. $129-131{ }^{\circ} \mathrm{C} .{ }^{1} \mathrm{H}$ NMR $\left(400 \mathrm{MHz}, \mathrm{CDCl}_{3}\right) \delta$ 7.96-7.91 (m, 2H), $7.60(\mathrm{~s}, 1 \mathrm{H}), 7.47-7.38(\mathrm{~m}, 3 \mathrm{H}), 7.29-7.23(\mathrm{~m}, 1 \mathrm{H}), 7.22-7.08(\mathrm{~m}, 3 \mathrm{H}), 3.02$ $-2.82(\mathrm{~m}, 2 \mathrm{H}), 2.24-2.12(\mathrm{~m}, 3 \mathrm{H}), 2.08-1.97(\mathrm{~m}, 1 \mathrm{H}) ;{ }^{13} \mathrm{C} \mathrm{NMR}\left(100 \mathrm{MHz}, \mathrm{CDCl}_{3}\right)$ $\delta 171.0,151.5,137.4,131.5,129.8,129.3,129.0,128.9,128.5,128.2,126.7$, 126.6, 126.0, 84.0, 34.1, 28.8, 19.4. FT-IR: $v\left(\mathrm{~cm}^{-1}\right) 3072,2954,1744,1575,1425,1339$. HRMS [ESI] calcd for $\mathrm{C}_{19} \mathrm{H}_{17} \mathrm{O}_{2}[\mathrm{M}+\mathrm{H}]^{+}$277.1229, found 277.1227; ESI $[\mathrm{M}+\mathrm{H}]^{+} 277.1$.

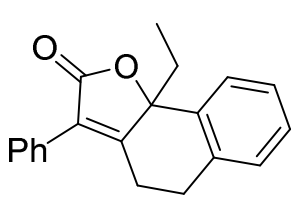

5c: yellow solid, m.p. $113-115{ }^{\circ} \mathrm{C} .{ }^{1} \mathrm{H}$ NMR $\left(400 \mathrm{MHz}, \mathrm{CDCl}_{3}\right) \delta$ 7.61-7.58 $(\mathrm{m}, 1 \mathrm{H}), 7.54-7.51(\mathrm{~m}, 2 \mathrm{H}), 7.45-7.40(\mathrm{~m}, 2 \mathrm{H}), 7.39-7.33(\mathrm{~m}, 1 \mathrm{H}), 7.33-7.23$ $(\mathrm{m}, 2 \mathrm{H}), 7.14(\mathrm{~d}, J=7.6 \mathrm{~Hz}, 1 \mathrm{H}), 3.26-3.15(\mathrm{~m}, 2 \mathrm{H}), 3.07-2.92(\mathrm{~m}, 2 \mathrm{H}), 2.10$ $(\mathrm{qd}, J=14.8,7.2 \mathrm{~Hz}, 1 \mathrm{H}), 1.94(\mathrm{qd}, J=14.8,7.2 \mathrm{~Hz}, 1 \mathrm{H}), 0.94(\mathrm{t}, J=7.6 \mathrm{~Hz}, 3 \mathrm{H}) ;{ }^{13} \mathrm{C}$ NMR $(100$ $\left.\mathrm{MHz}, \mathrm{CDCl}_{3}\right) \delta 171.3,163.4,137.6,133.5,129.5,128.3,128.0,127.8,127.6,126.5,125.2,124.7,86.1$, 32.0, 28.4, 22.0, 7.5. FT-IR: $v\left(\mathrm{~cm}^{-1}\right) 3054,2972,1739,1601,1458,1366$. HRMS [ESI] calcd for $\mathrm{C}_{20} \mathrm{H}_{18} \mathrm{O}_{2} \mathrm{Na}[\mathrm{M}+\mathrm{Na}]^{+}$313.1204, found 313.1191; ESI [M+H] ${ }^{+} 291.1$.

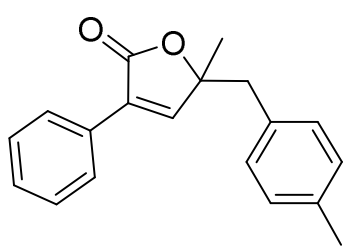

10: yellow solid, m.p. $75-77^{\circ} \mathrm{C} .{ }^{1} \mathrm{H}$ NMR (400 MHz, $\left.\mathrm{CDCl}_{3}\right) \delta$ 7.74-7.69 (m, 2H), $7.43(\mathrm{~s}, 1 \mathrm{H}), 7.39-7.33(\mathrm{~m}, 3 \mathrm{H}), 7.10-7.07$ (m, 4H), 3.07 (d, $J=$ $0.8 \mathrm{~Hz}, 2 \mathrm{H}), 2.29$ (s, 3H), $1.53(\mathrm{~s}, 3 \mathrm{H}) ;{ }^{13} \mathrm{C}$ NMR (100 MHz, $\left.\mathrm{CDCl}_{3}\right) \delta$ $170.4,151.4,136.3,131.2,130.6,129.7,129.2,128.7,128.6,128.1,126.6$, 85.1, 44.7, 23.4, 20.6. FT-IR: $v\left(\mathrm{~cm}^{-1}\right) 3055,2978,1748,1598,1447,1329$. HRMS [ESI] calcd for $\mathrm{C}_{19} \mathrm{H}_{18} \mathrm{O}_{2} \mathrm{Na}[\mathrm{M}+\mathrm{Na}]^{+}$301.1204, found 301.1210; ESI [M+H] ${ }^{+} 279.1$. 


\section{KIE experiment}
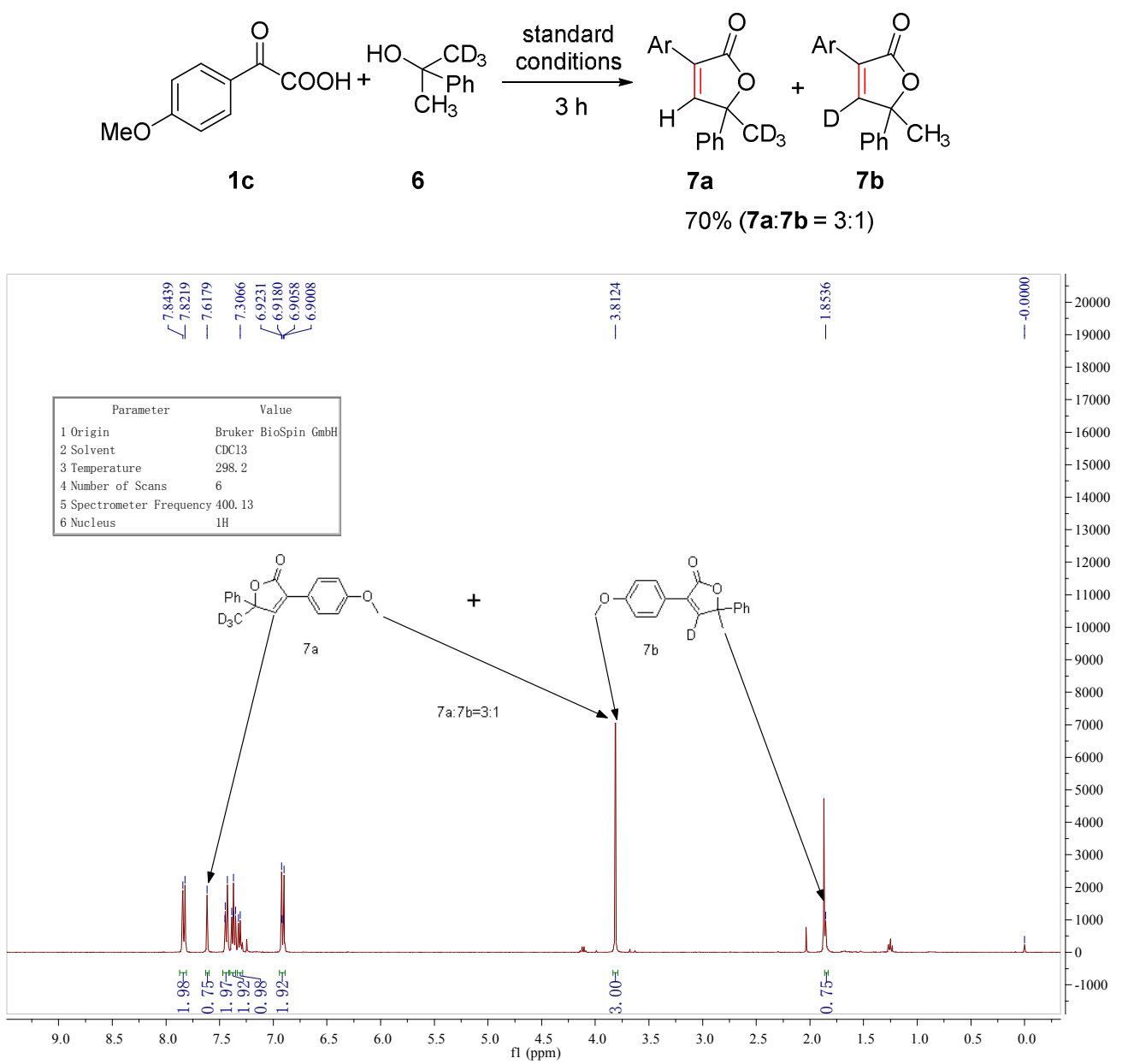
5. ${ }^{1} \mathrm{H},{ }^{13} \mathrm{C}$, and ${ }^{19} \mathrm{~F}$ NMR spectra of products
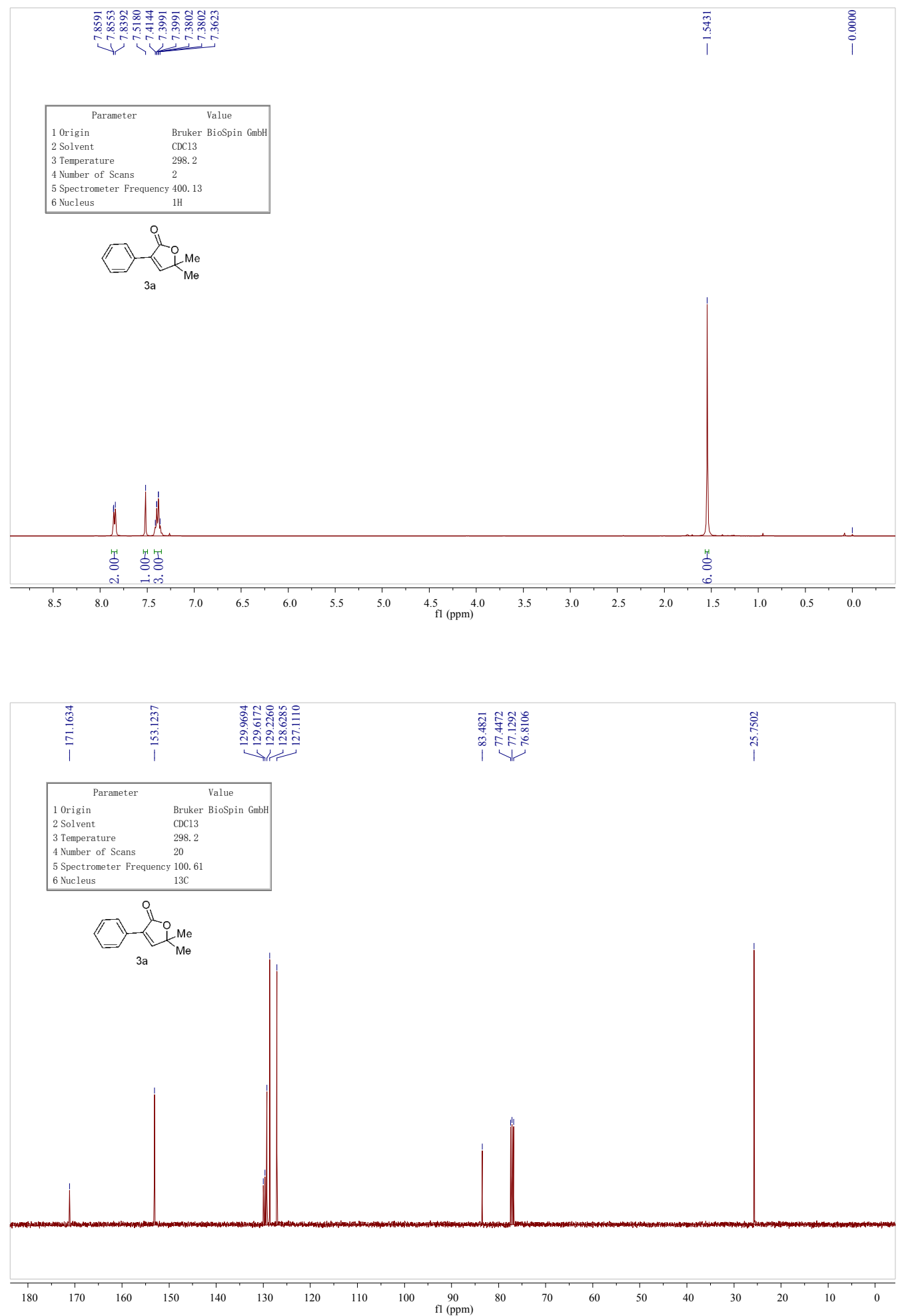

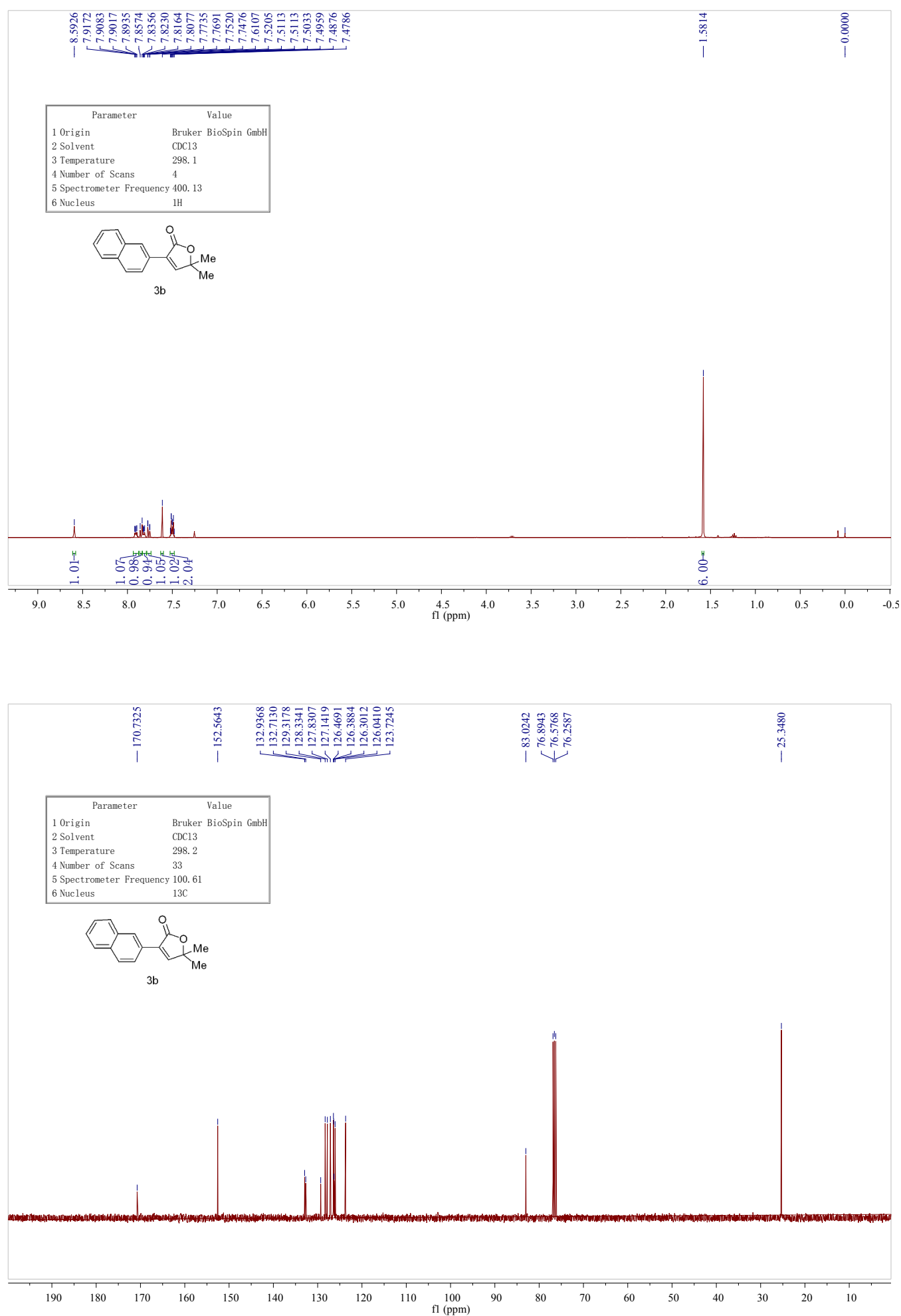

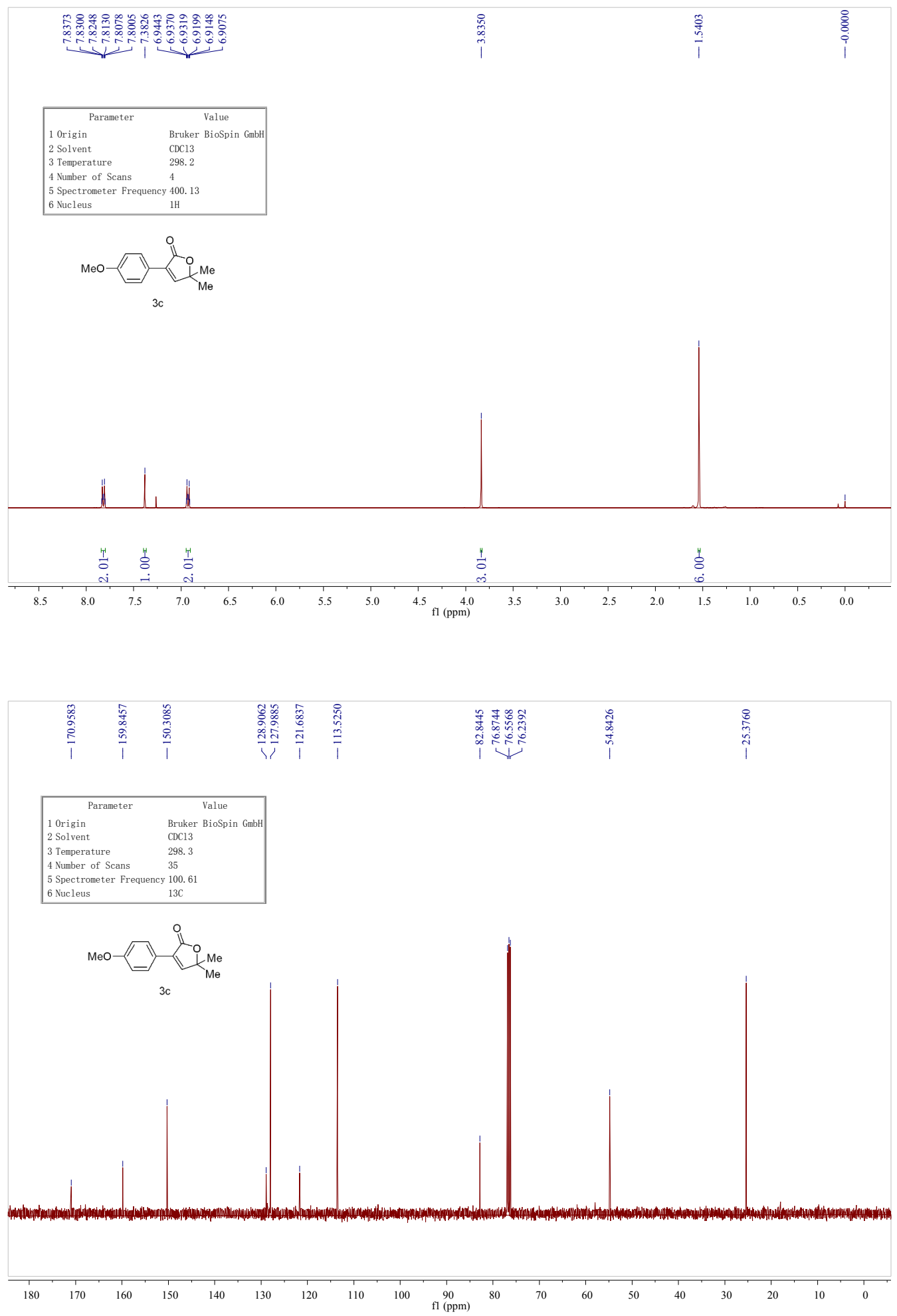

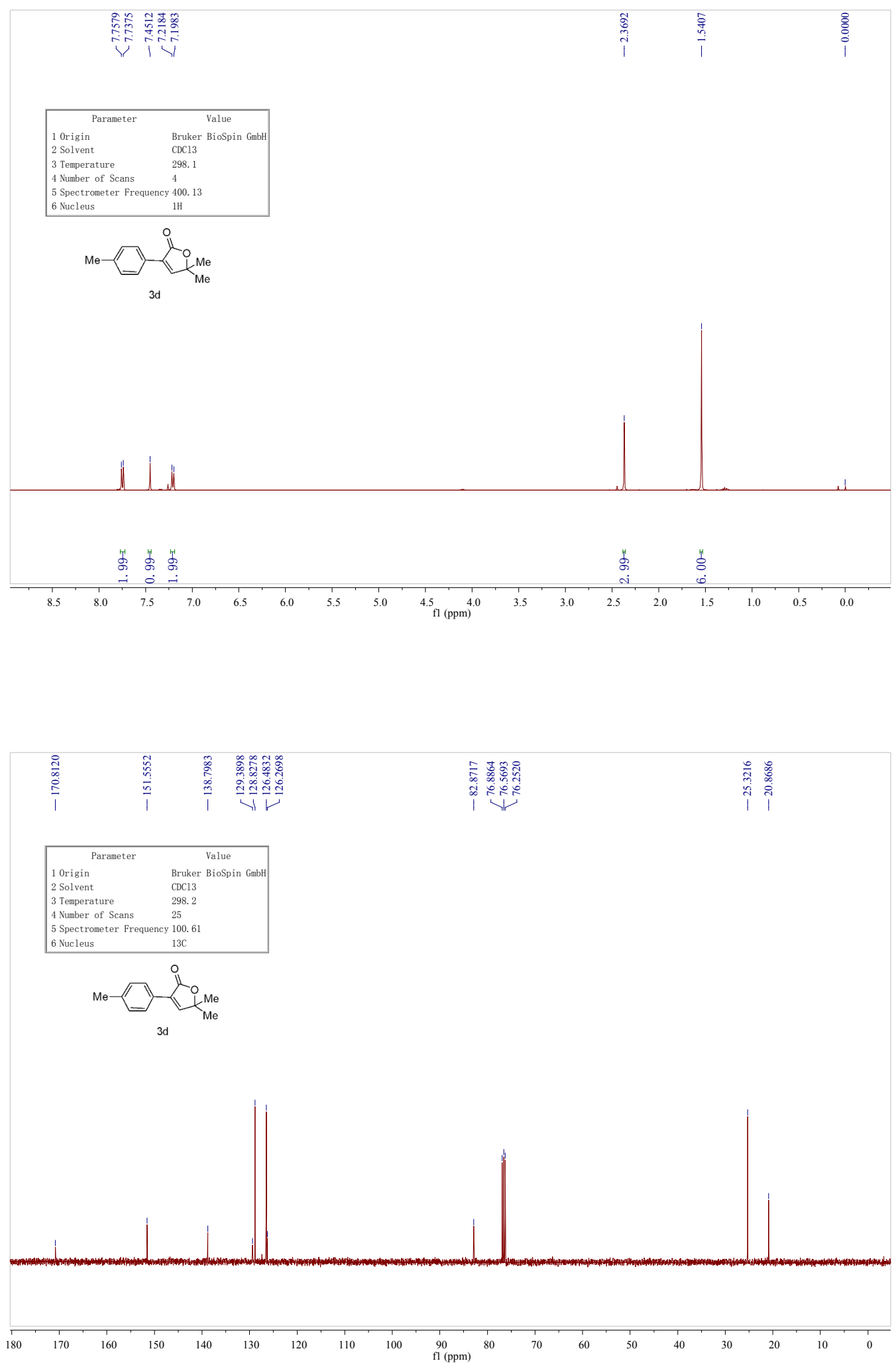

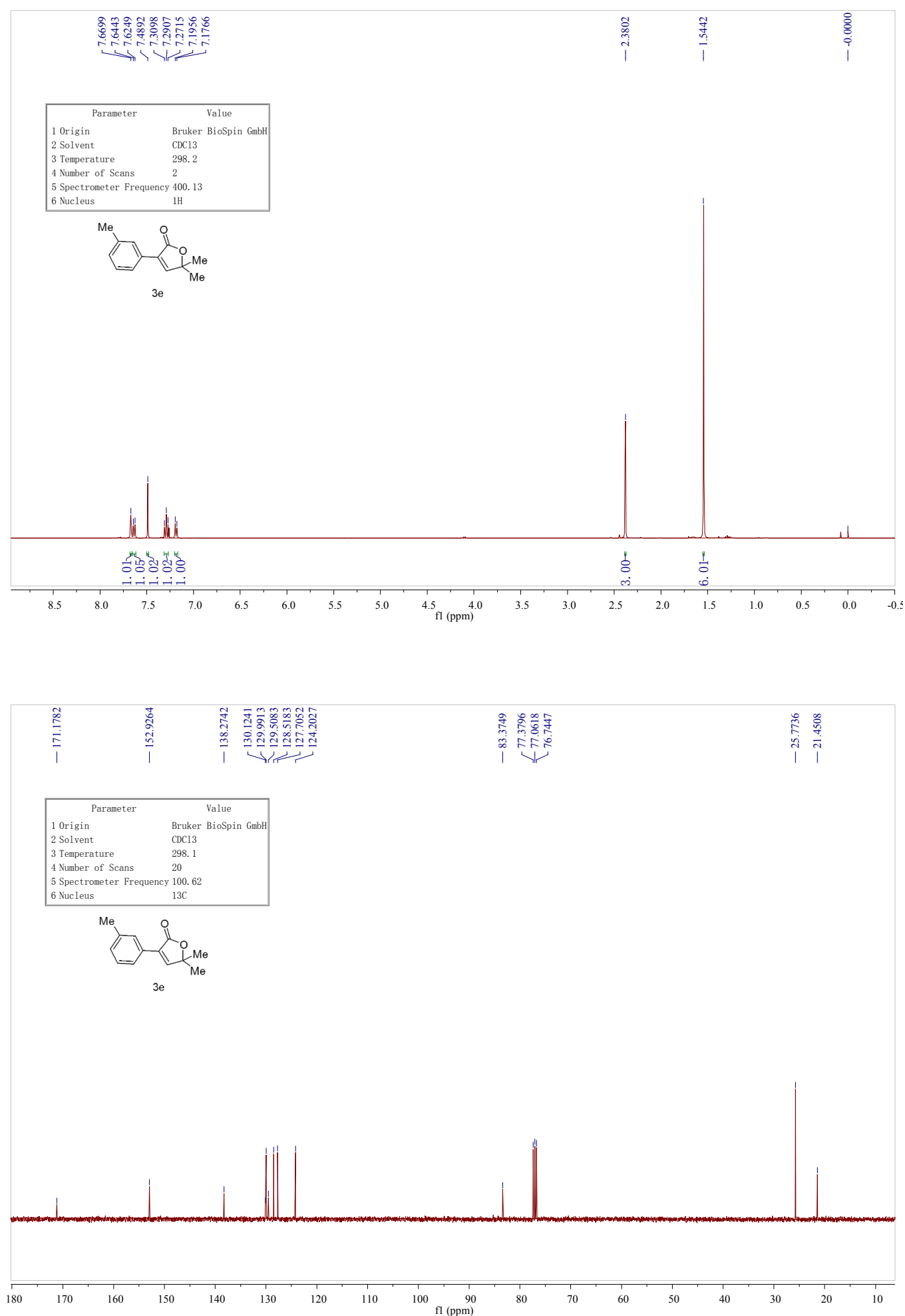

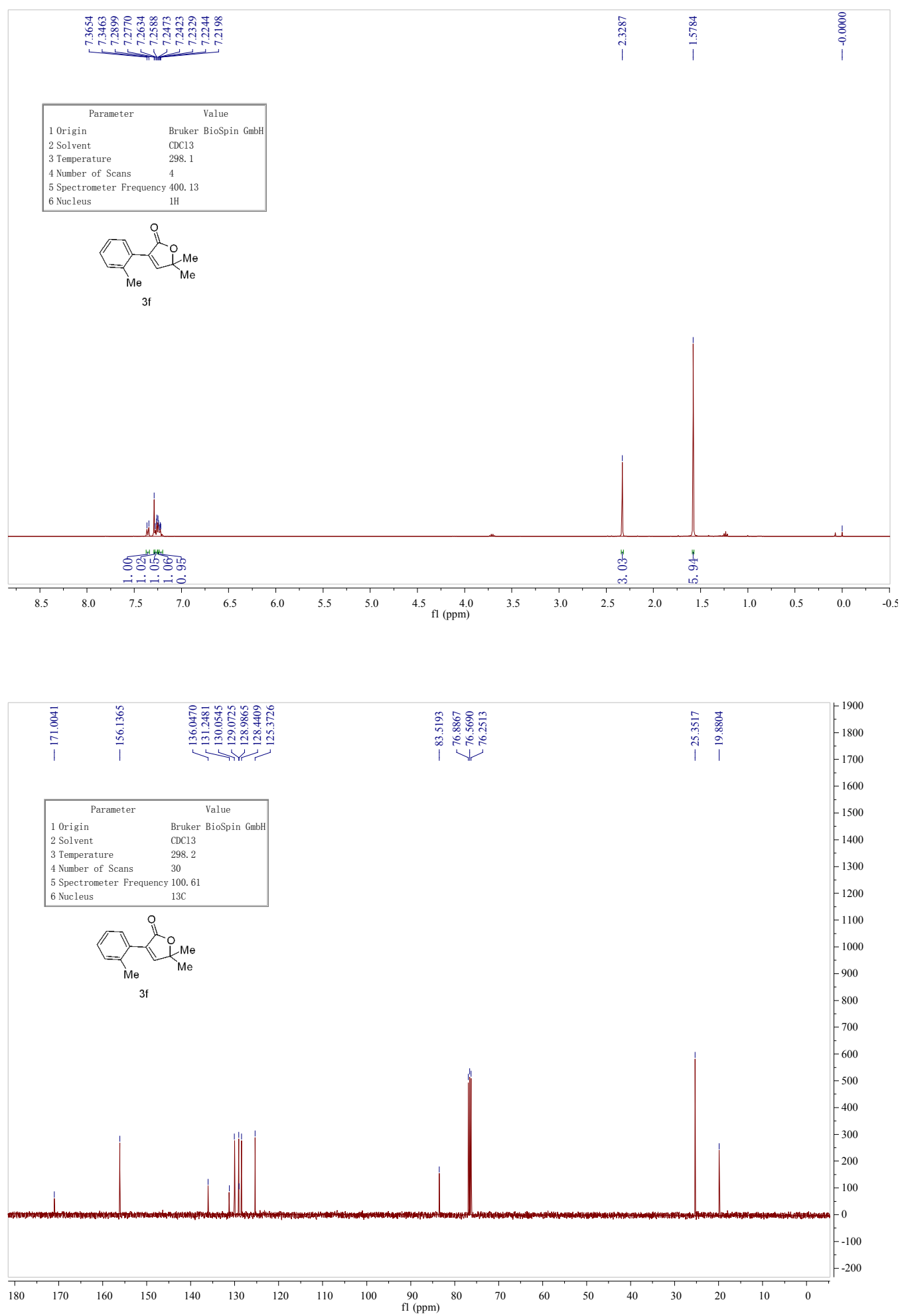

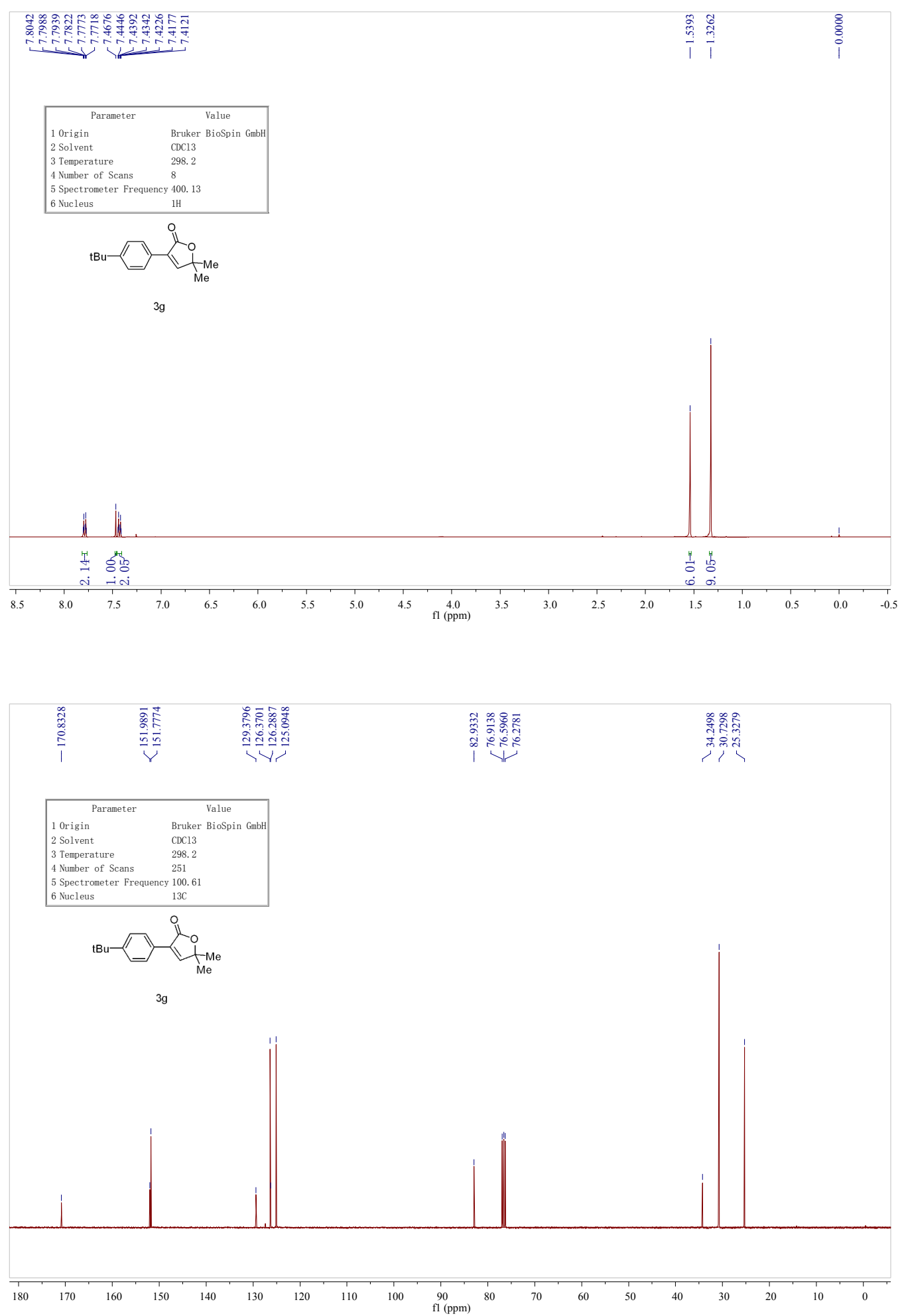

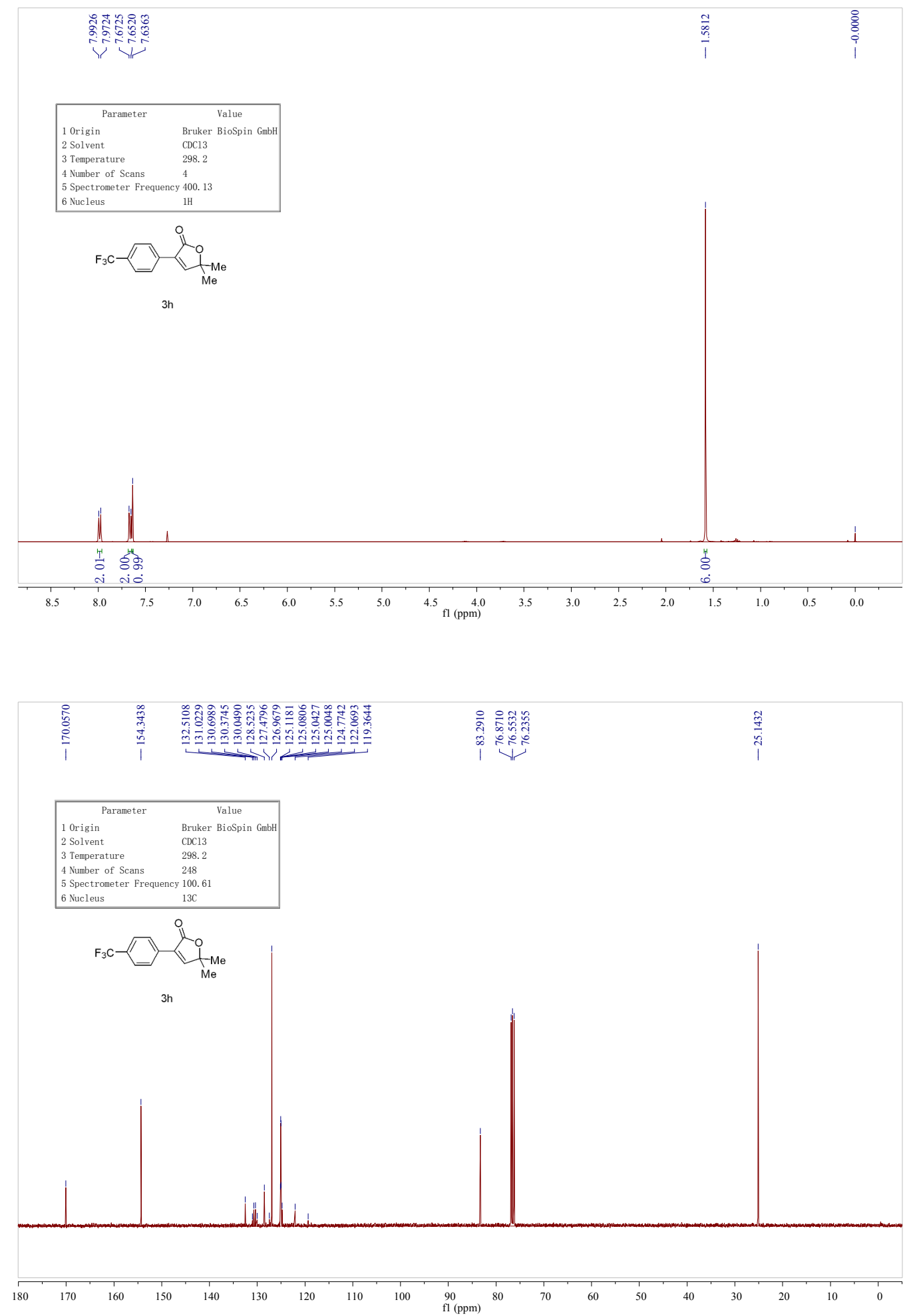

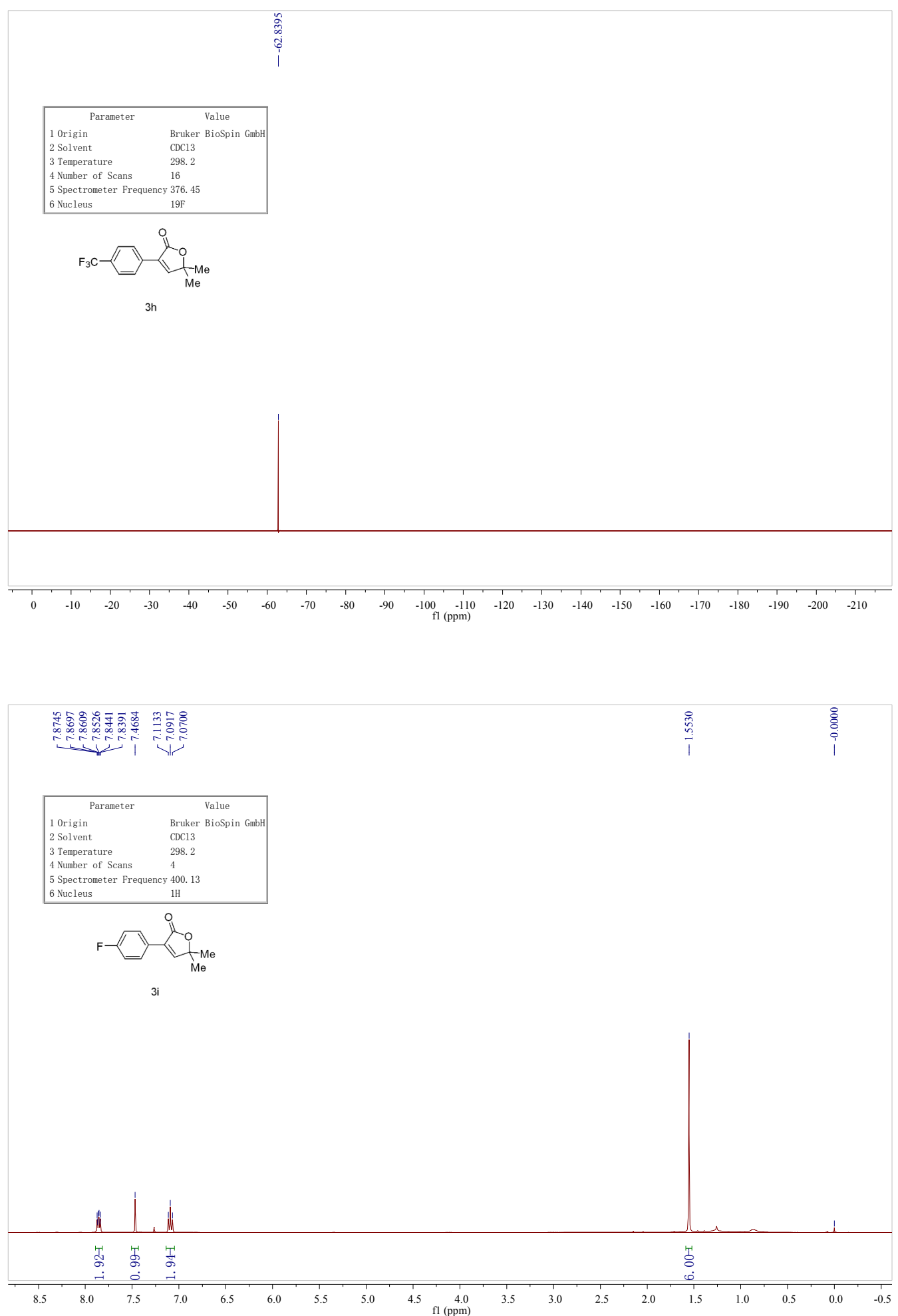

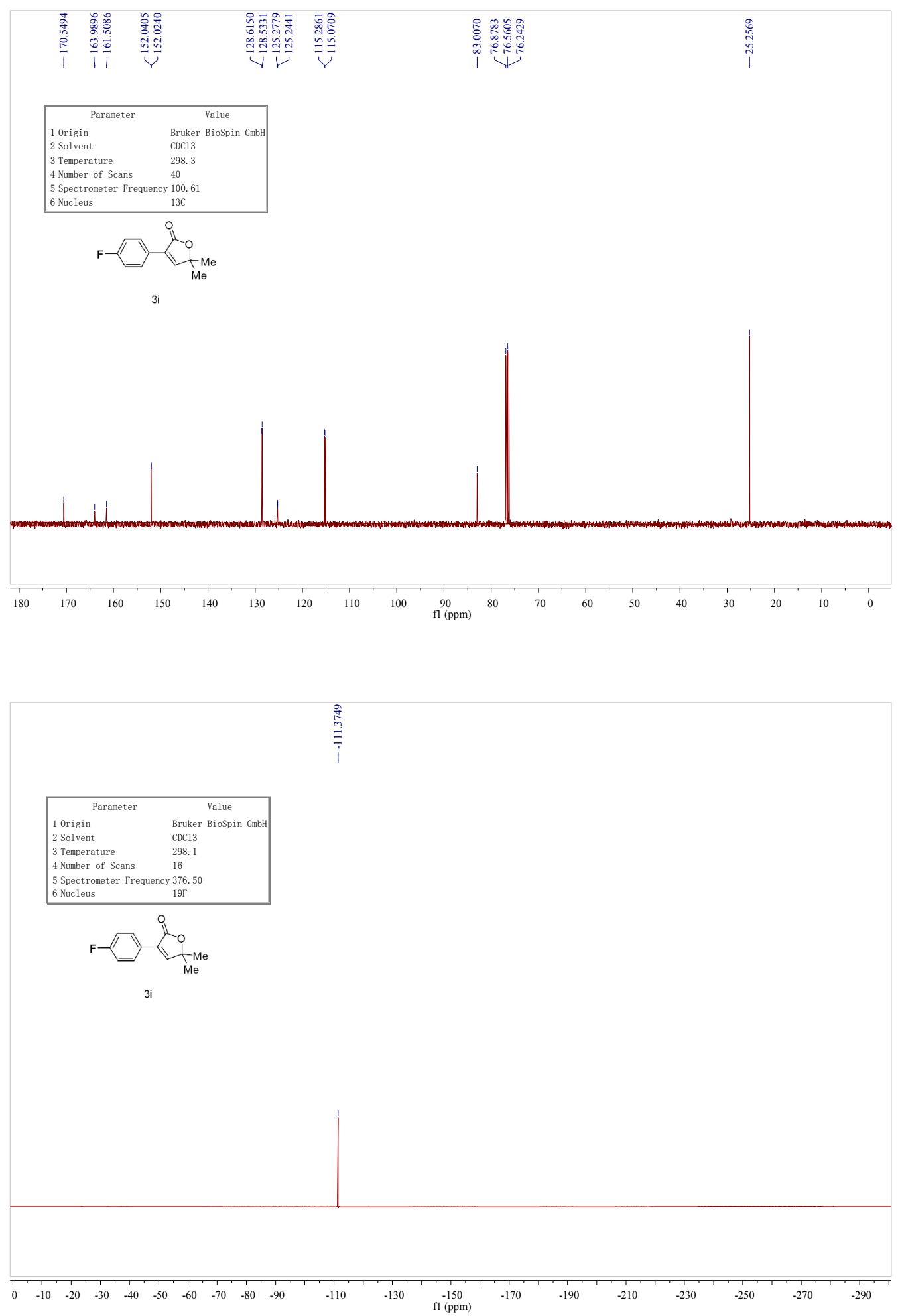

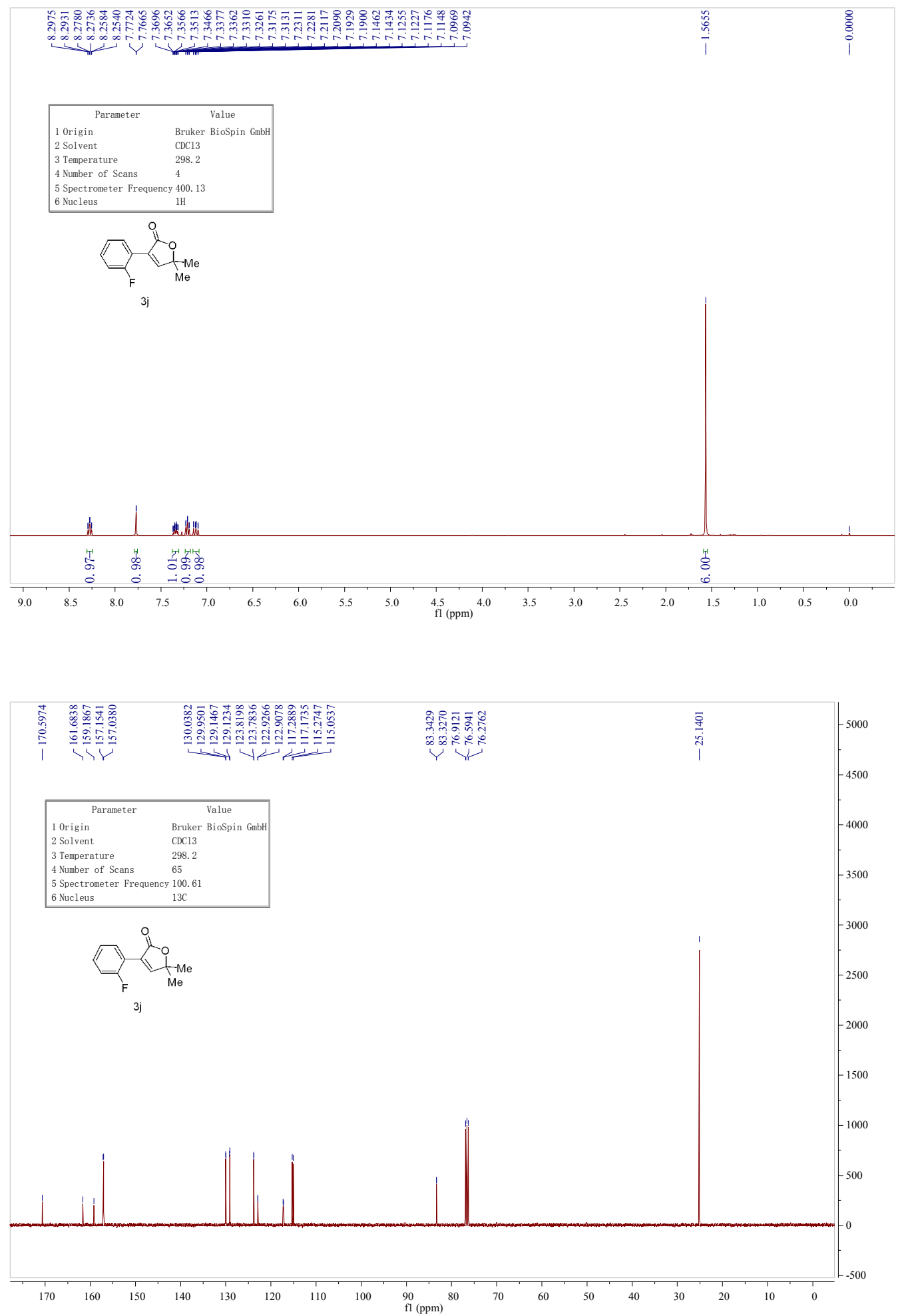

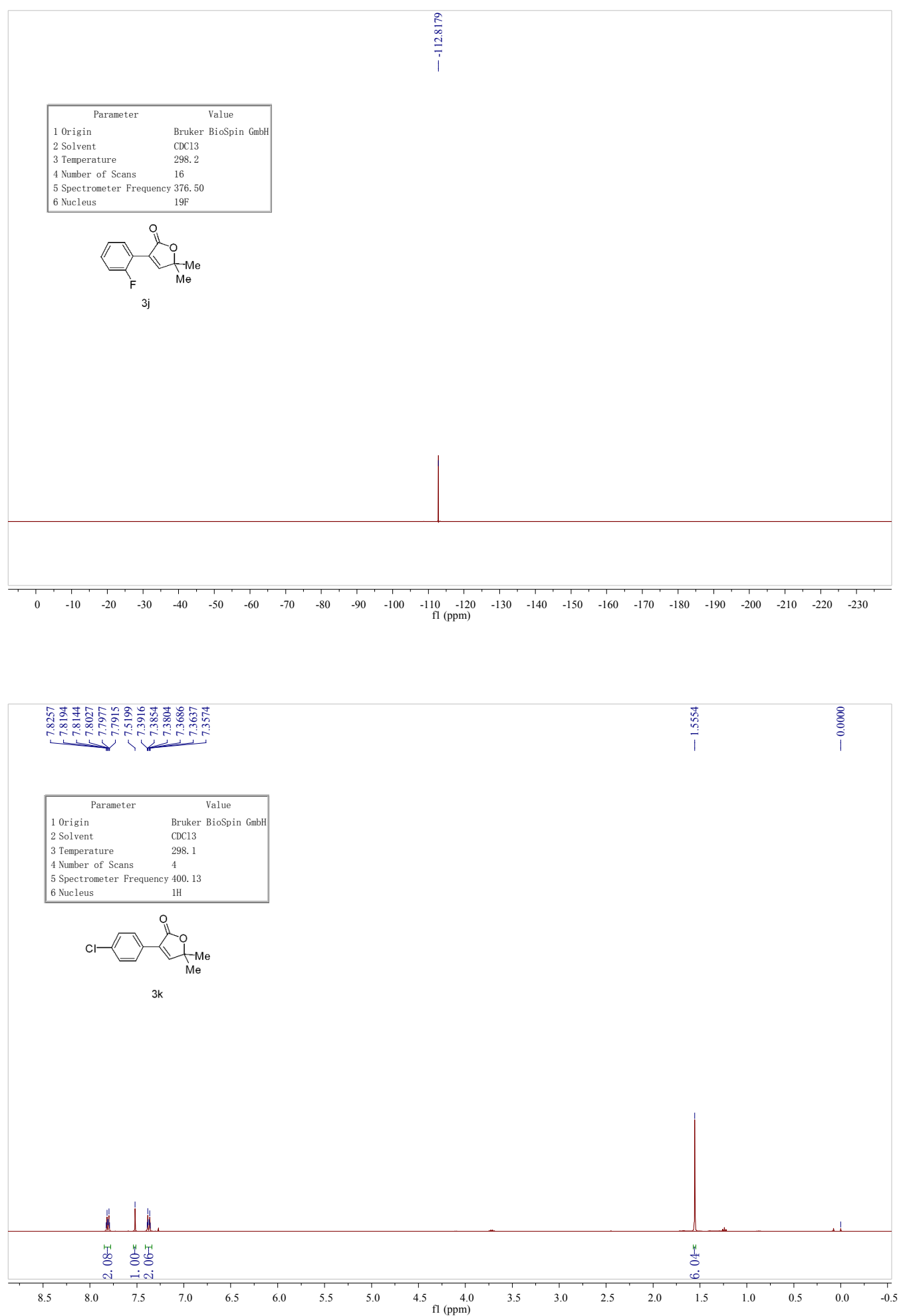

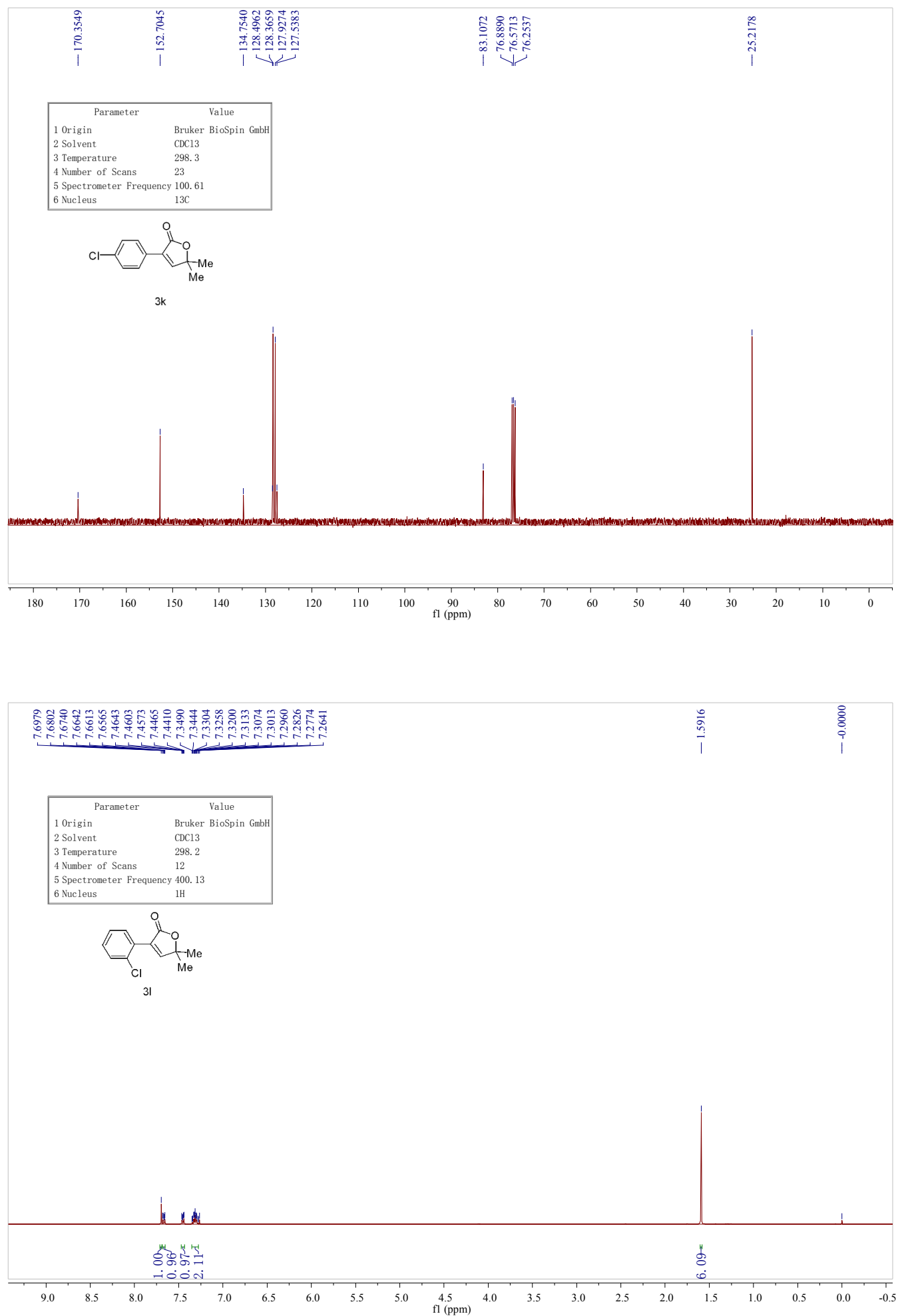

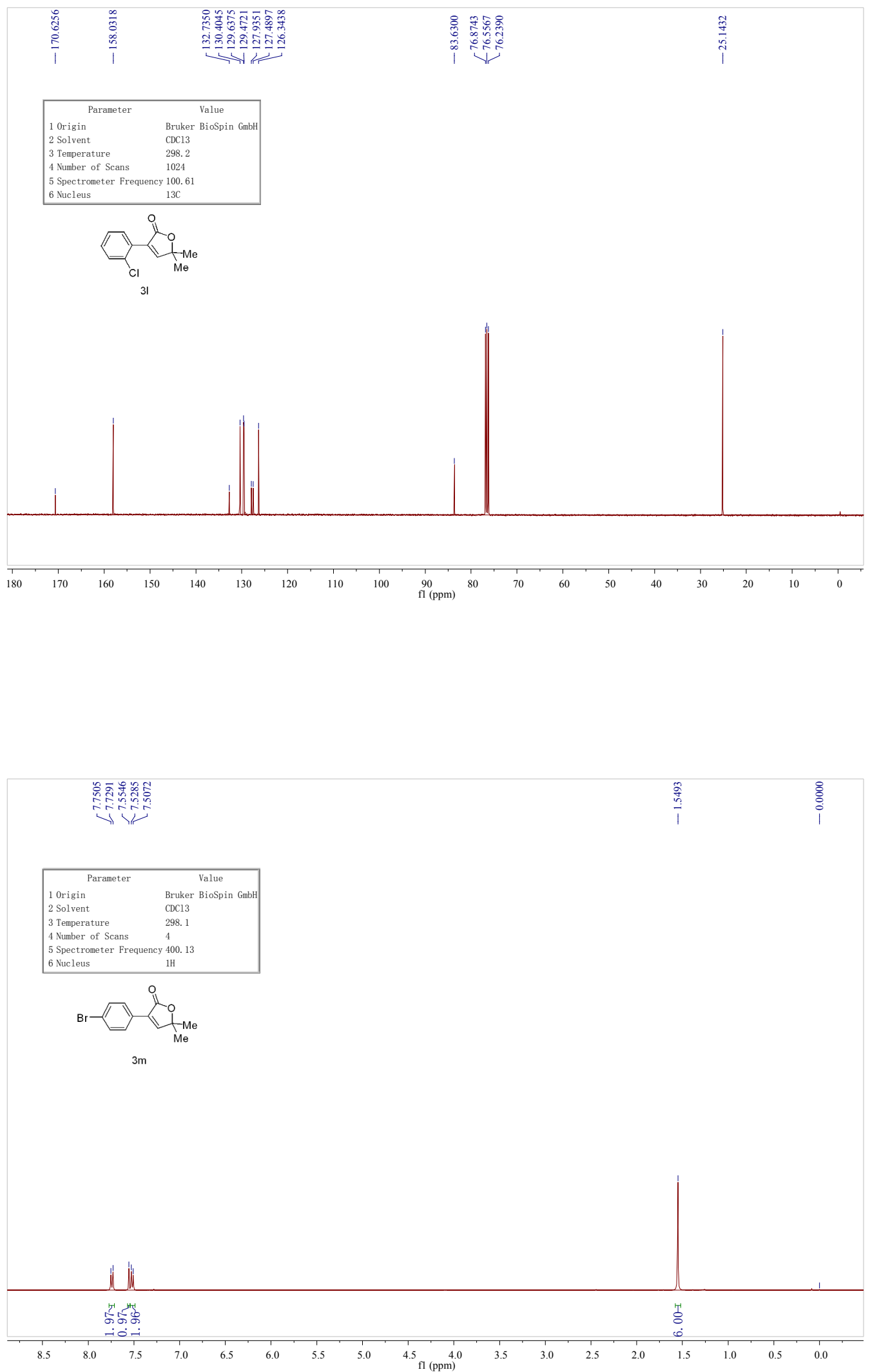

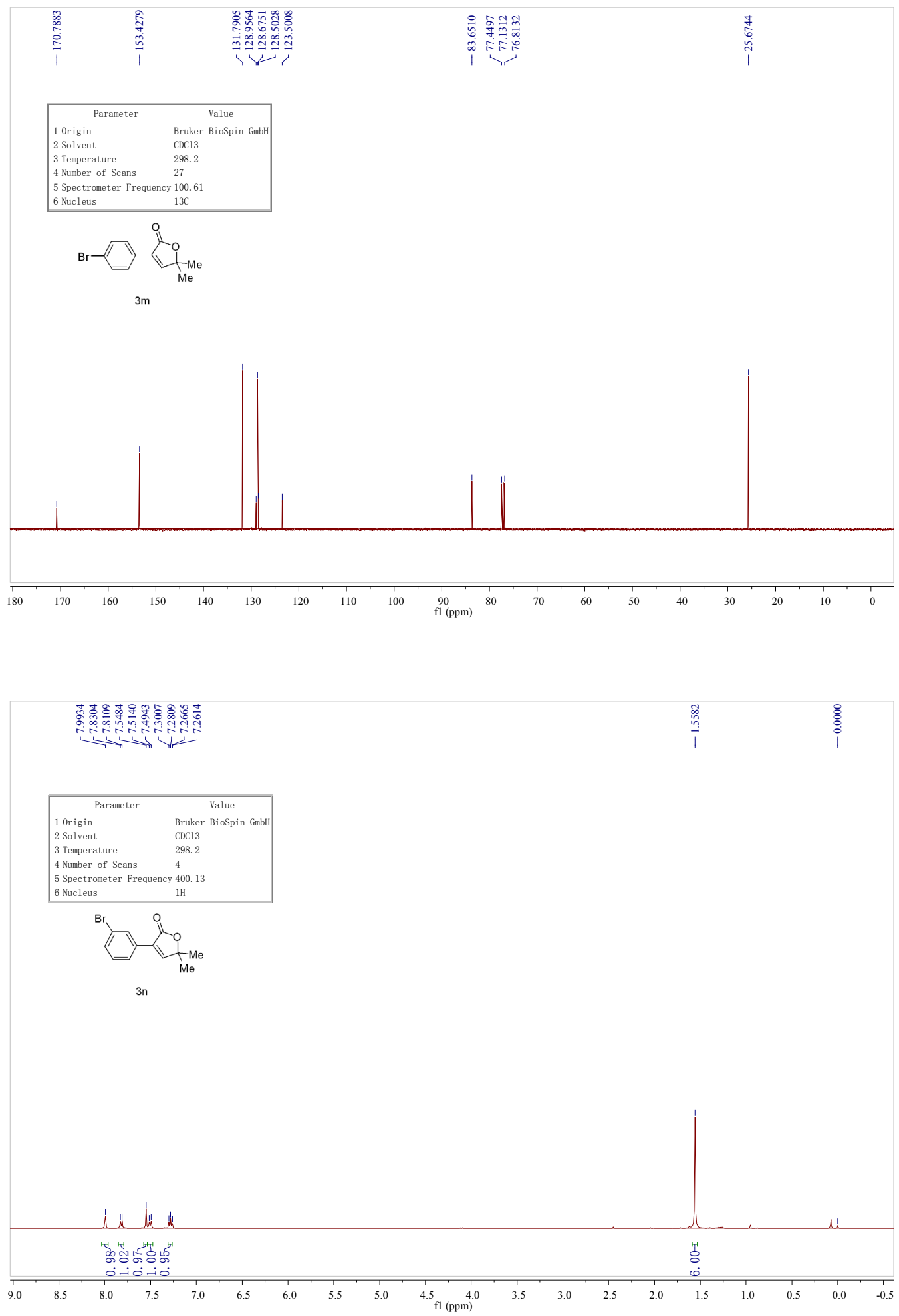

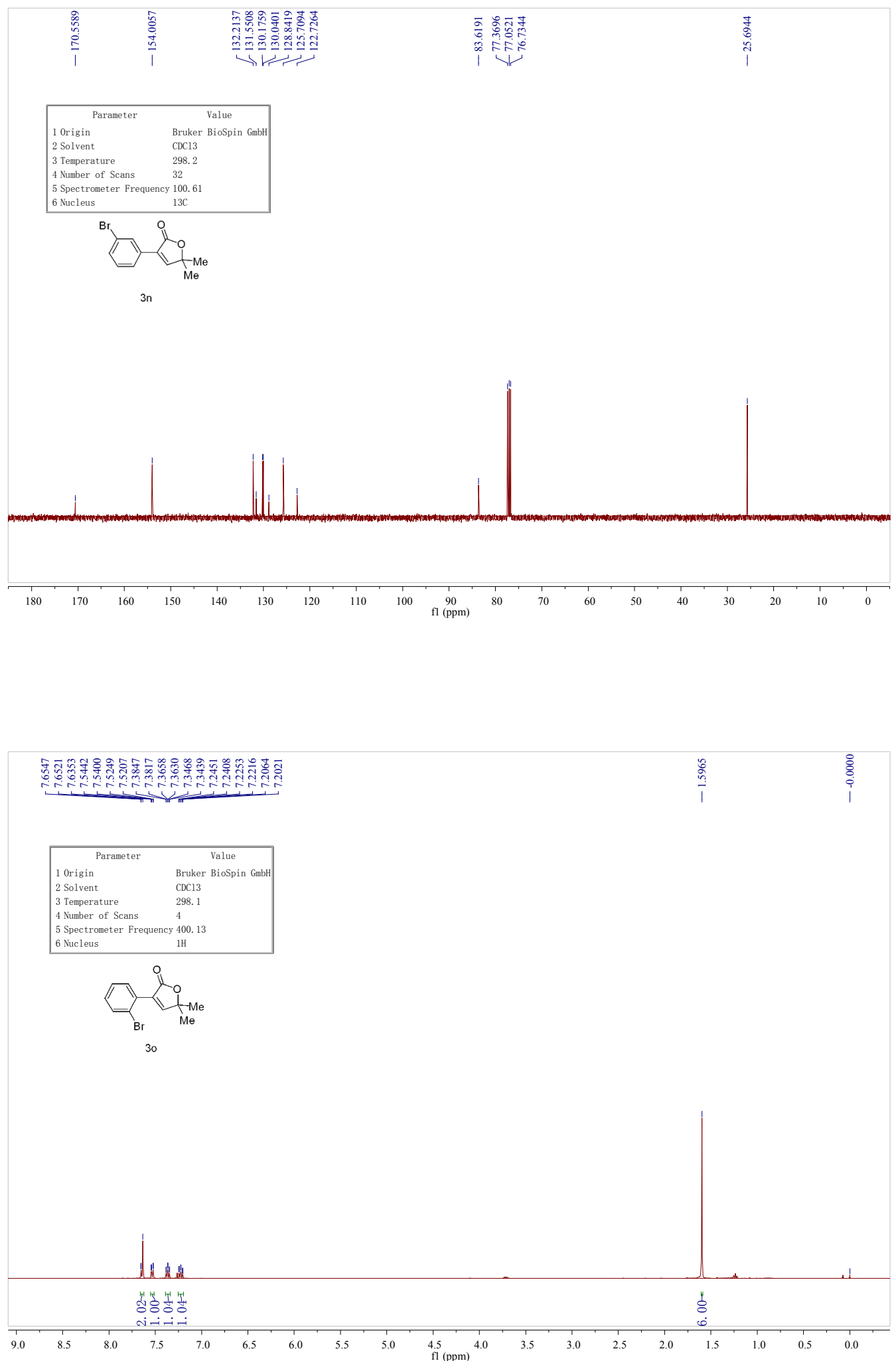

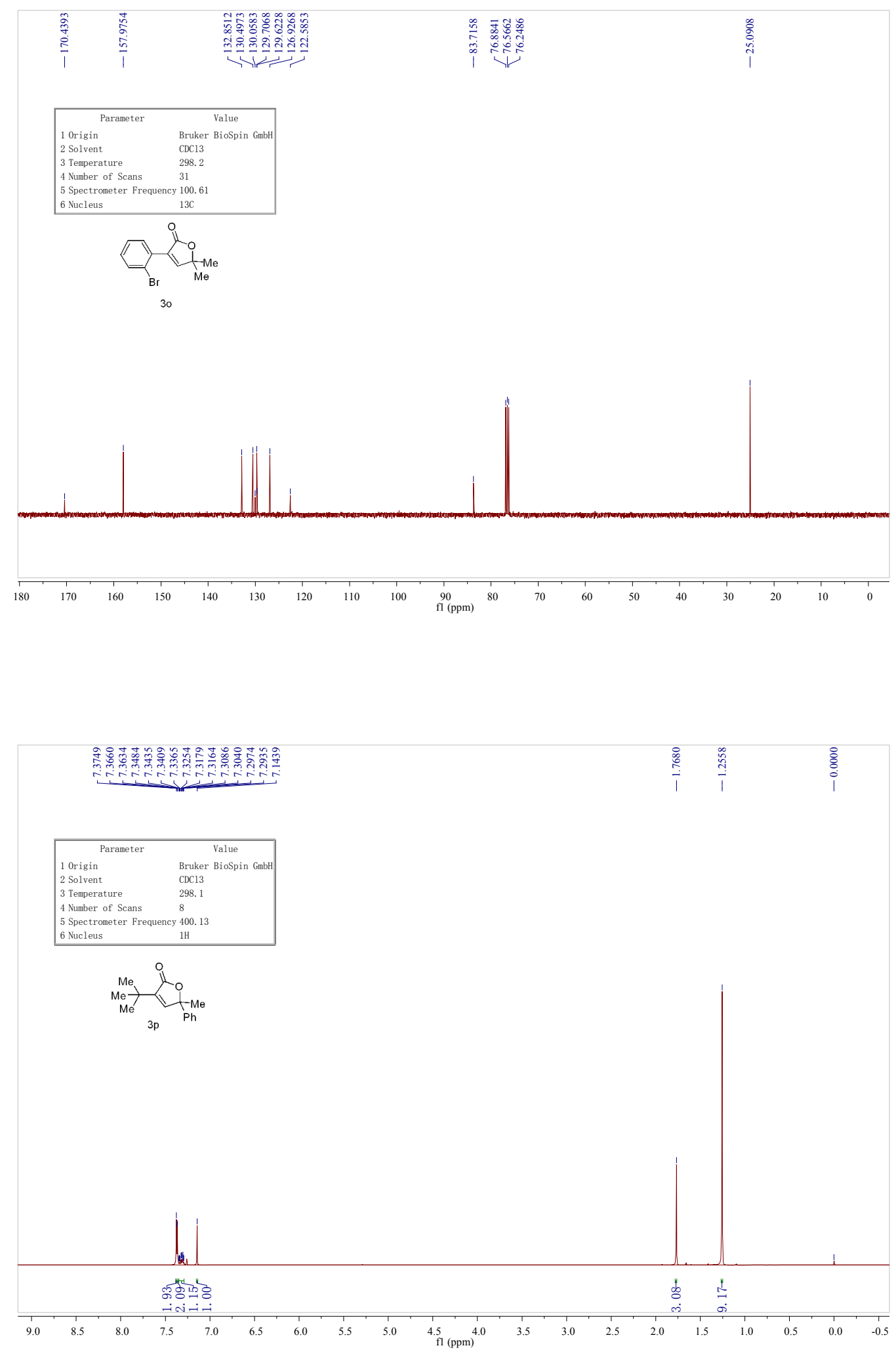

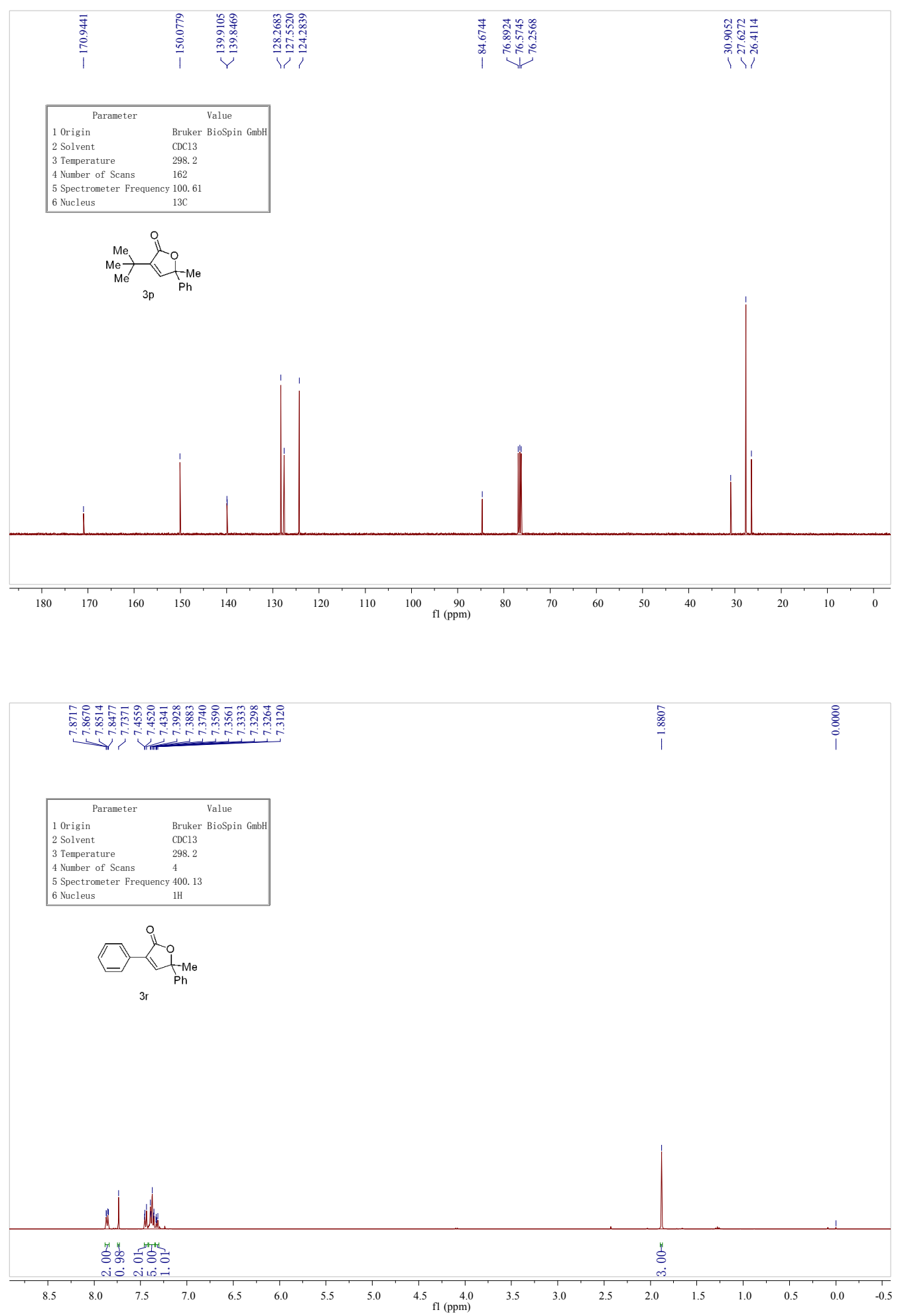

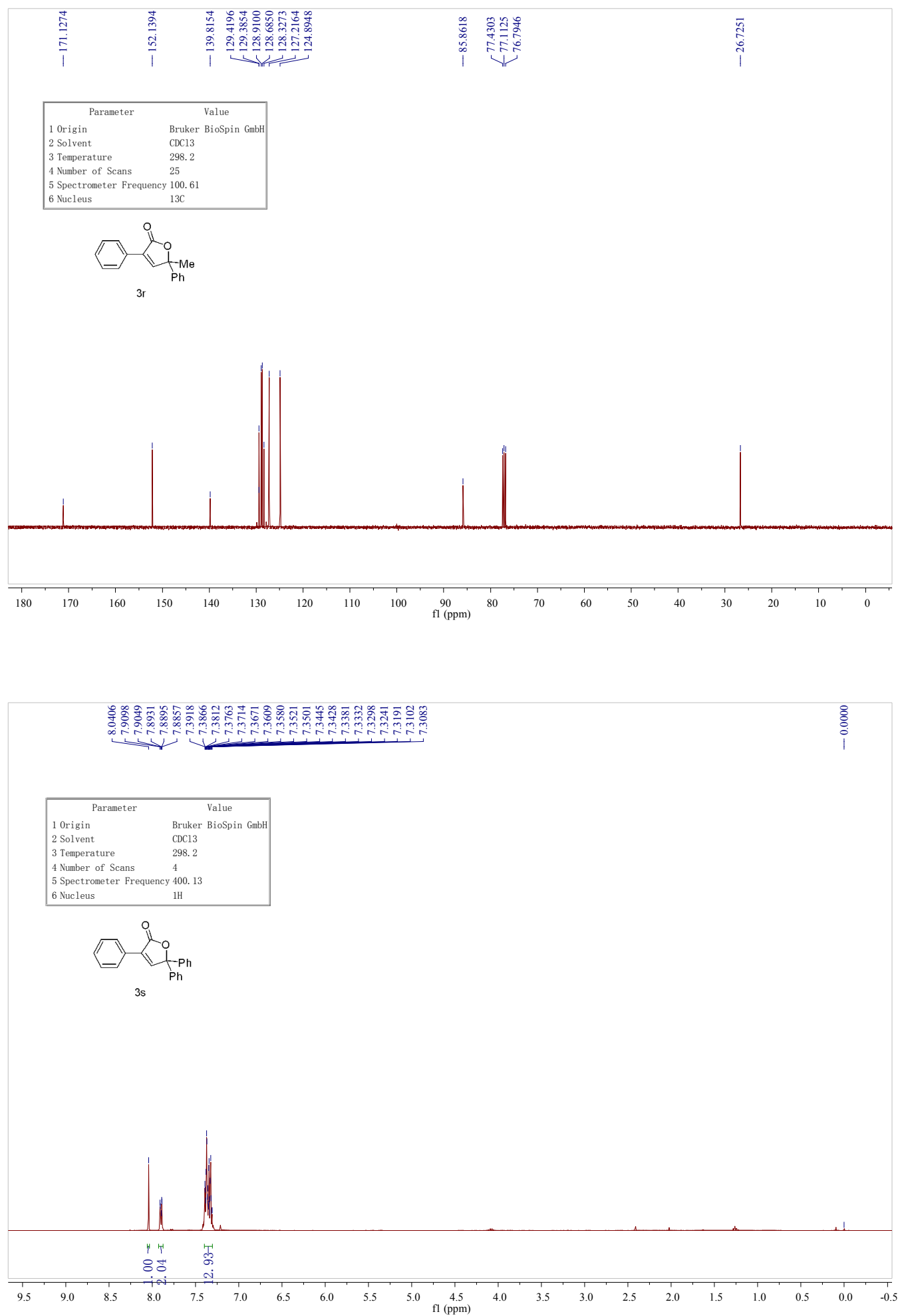

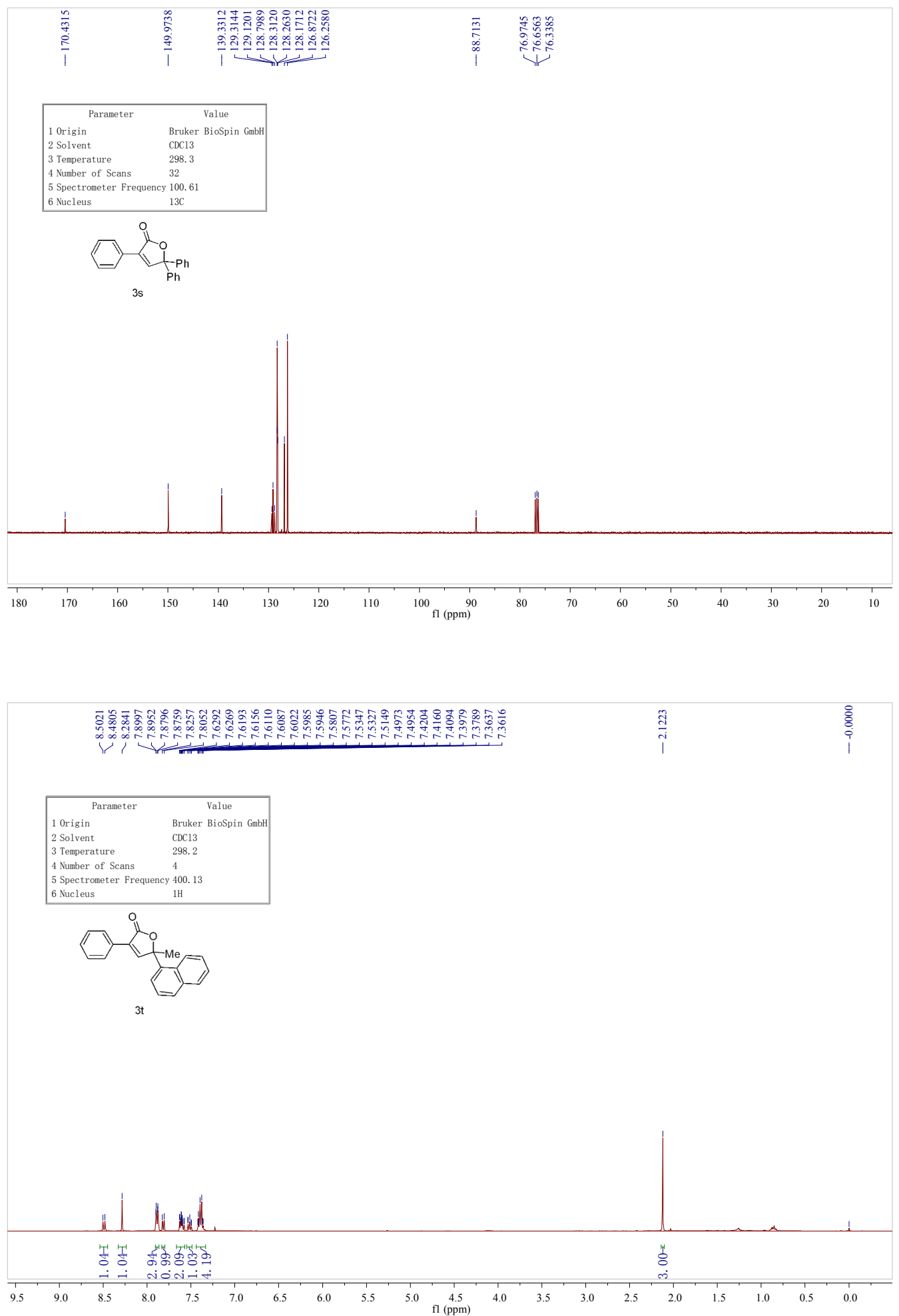

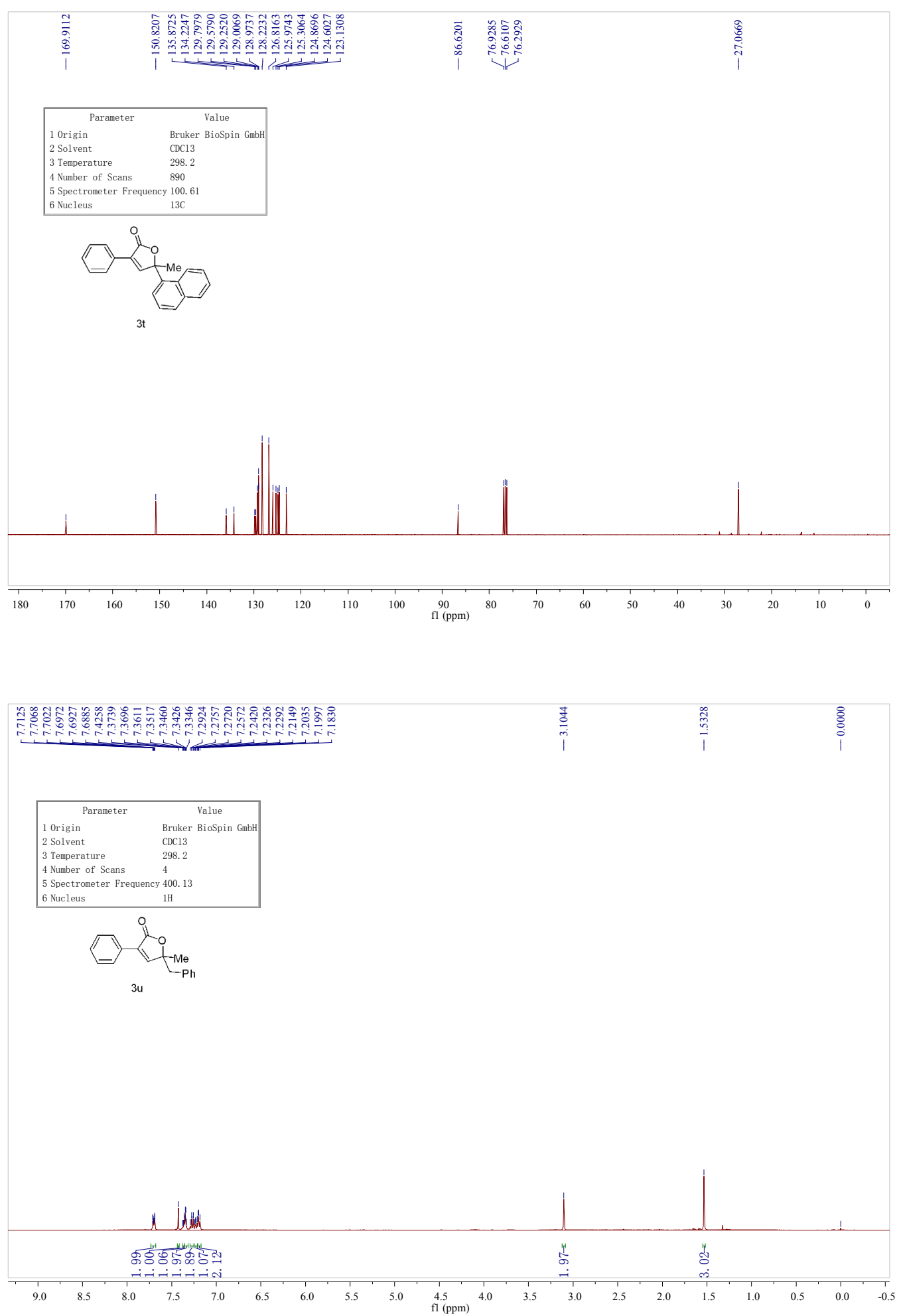

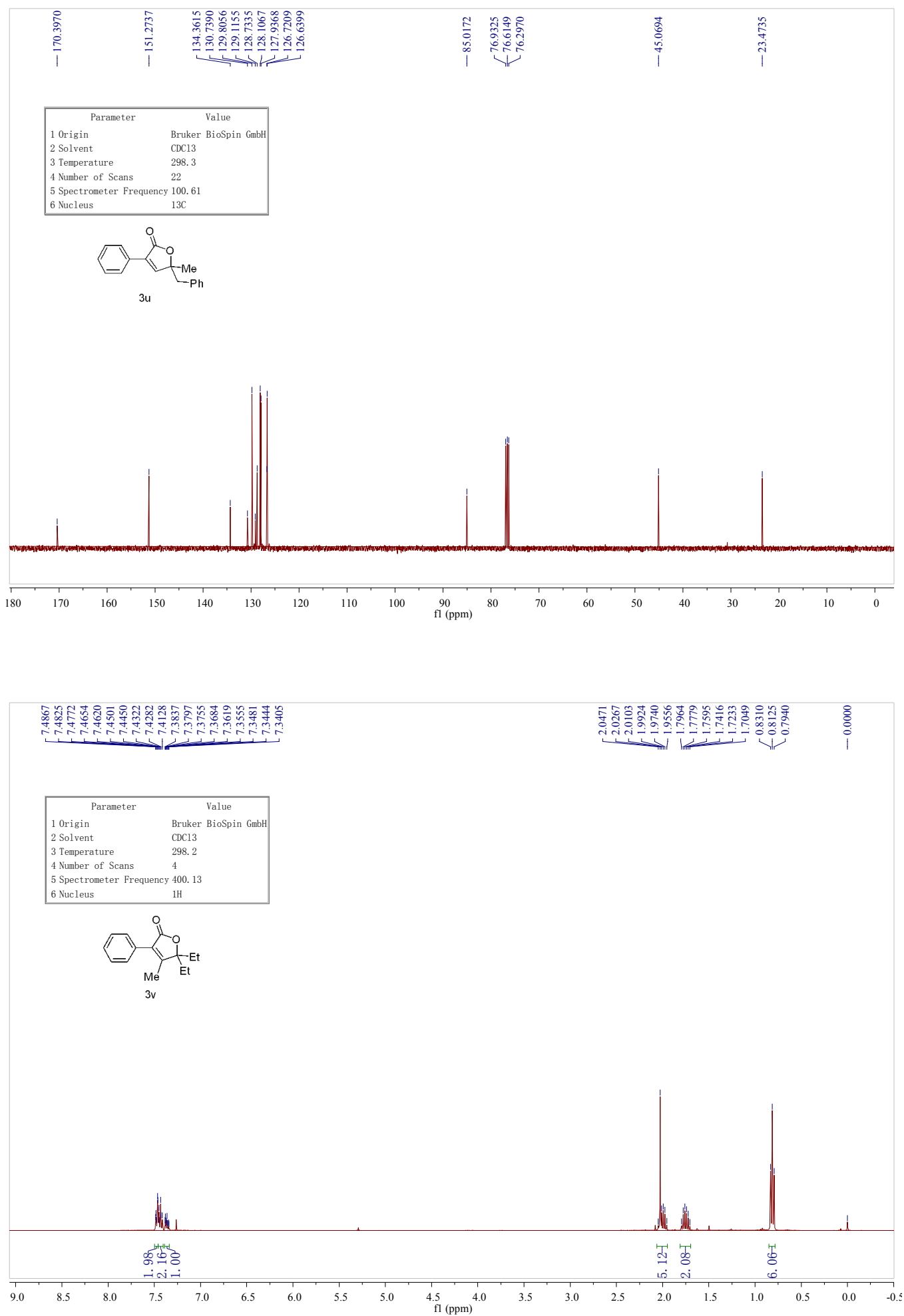

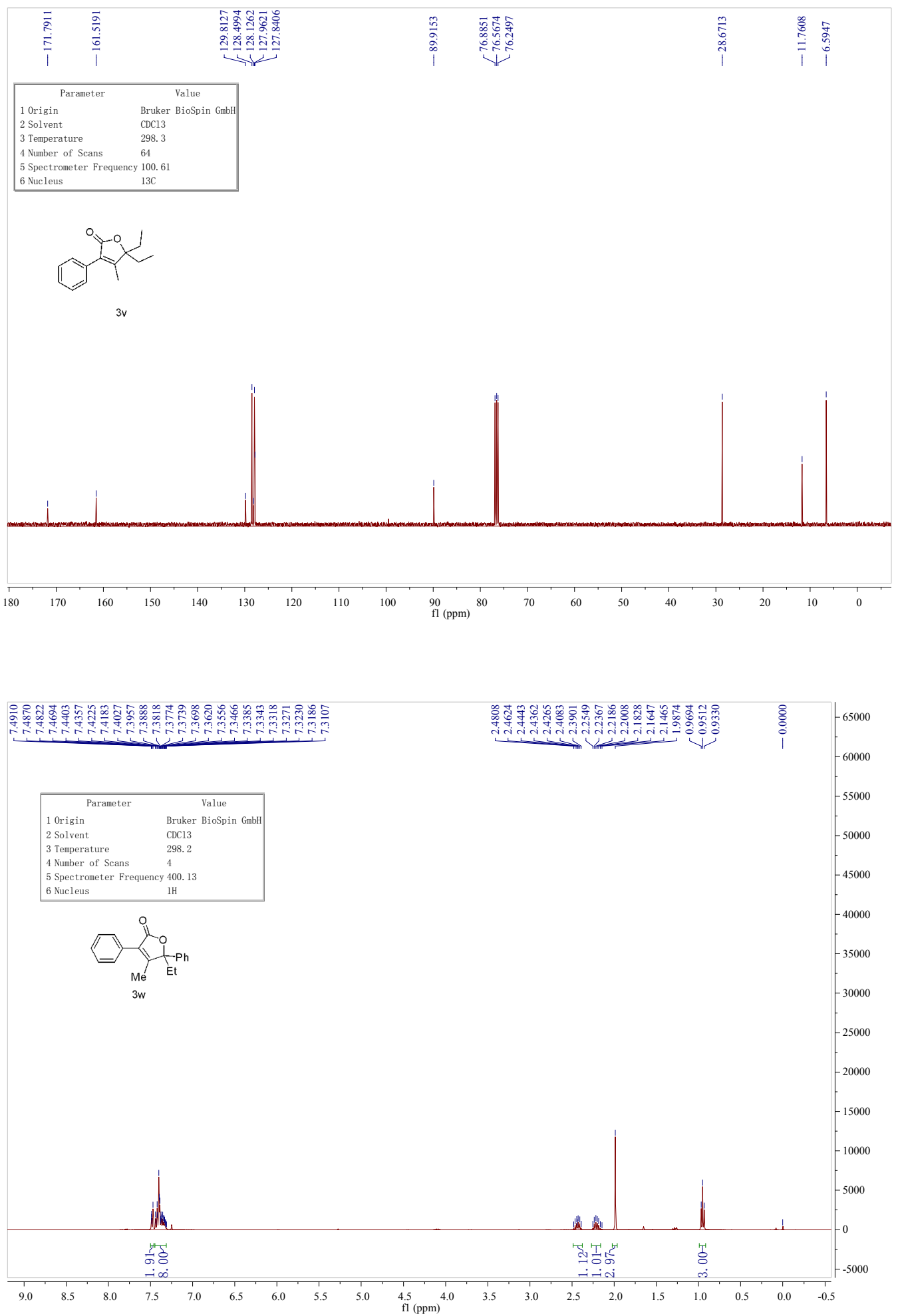

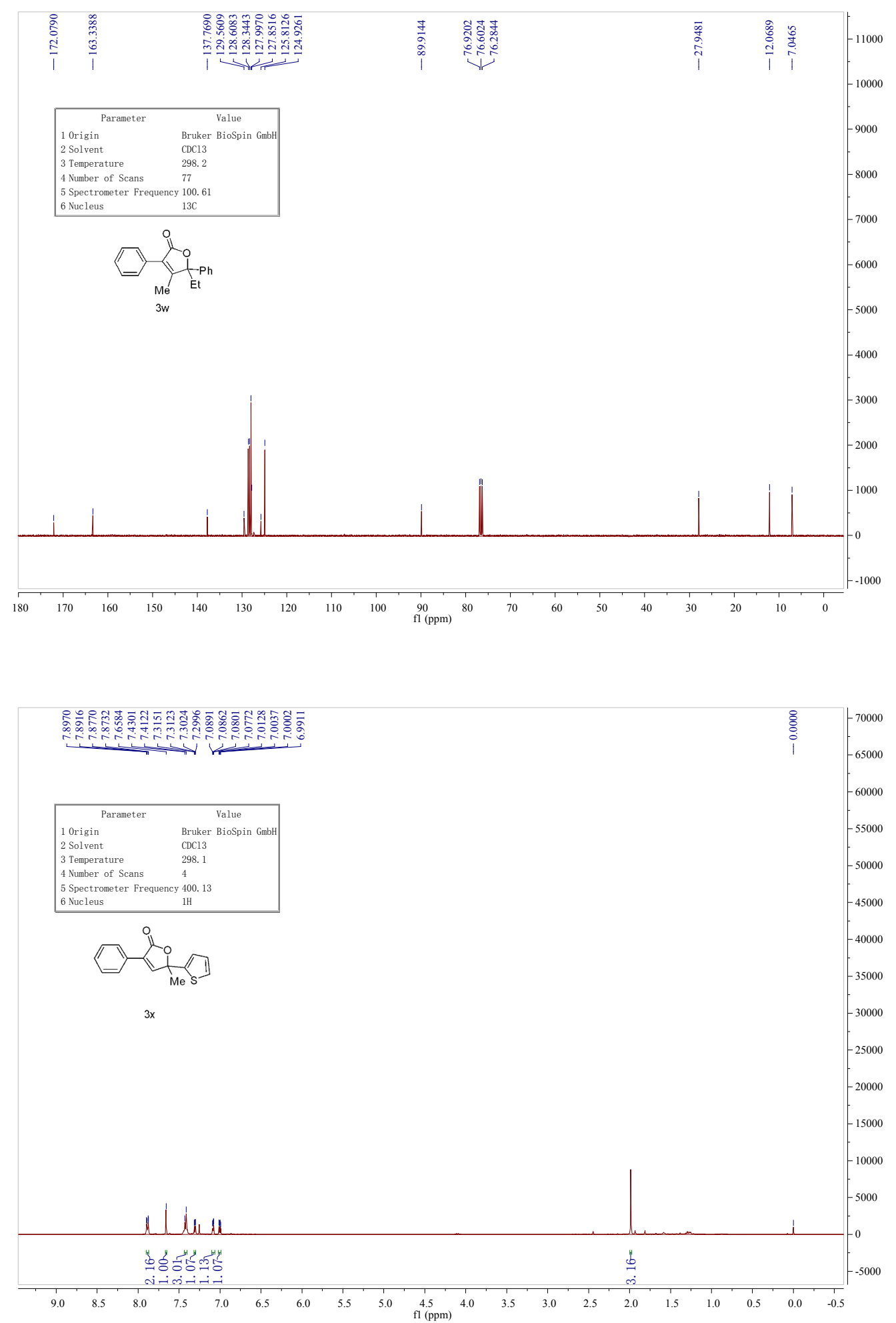

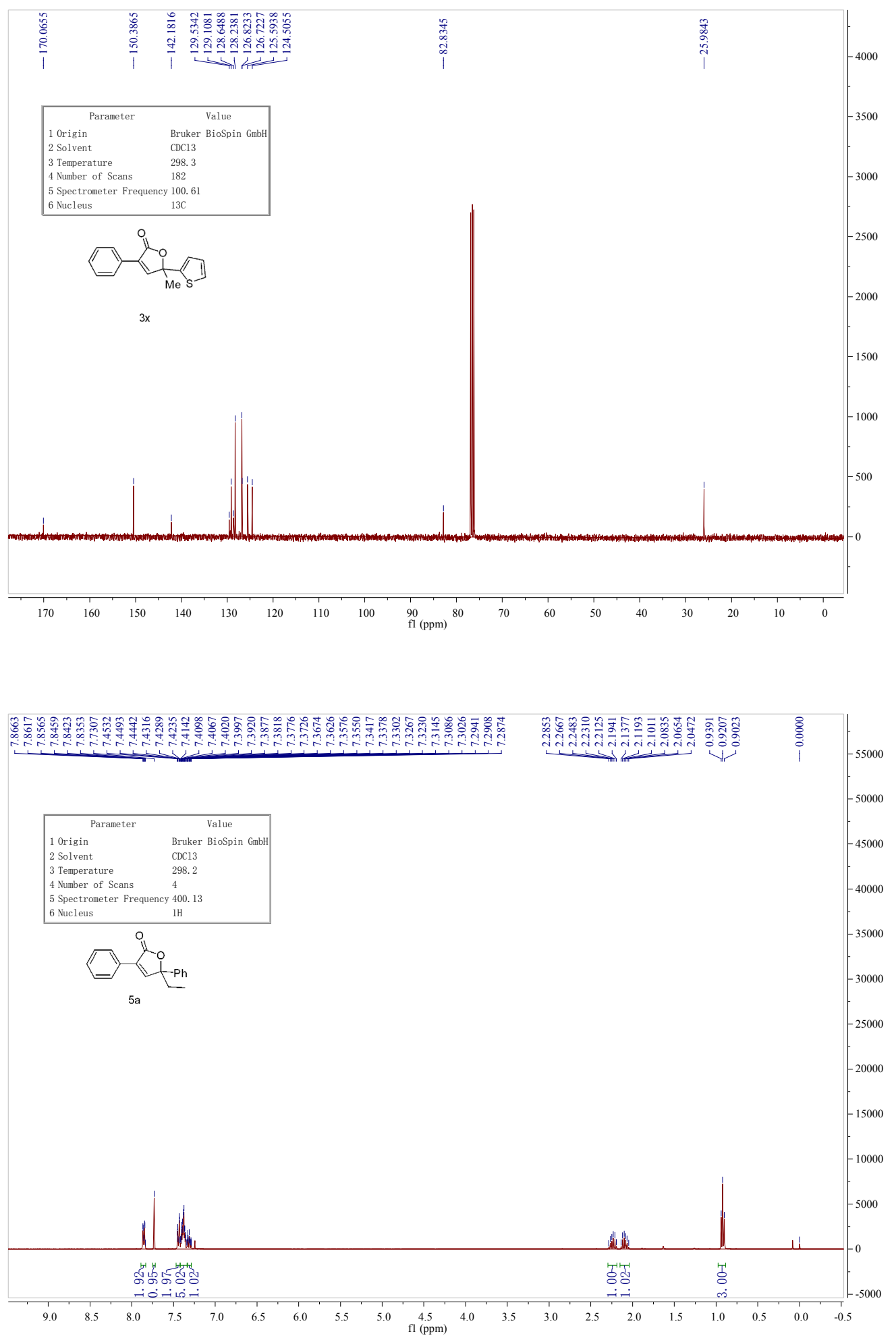

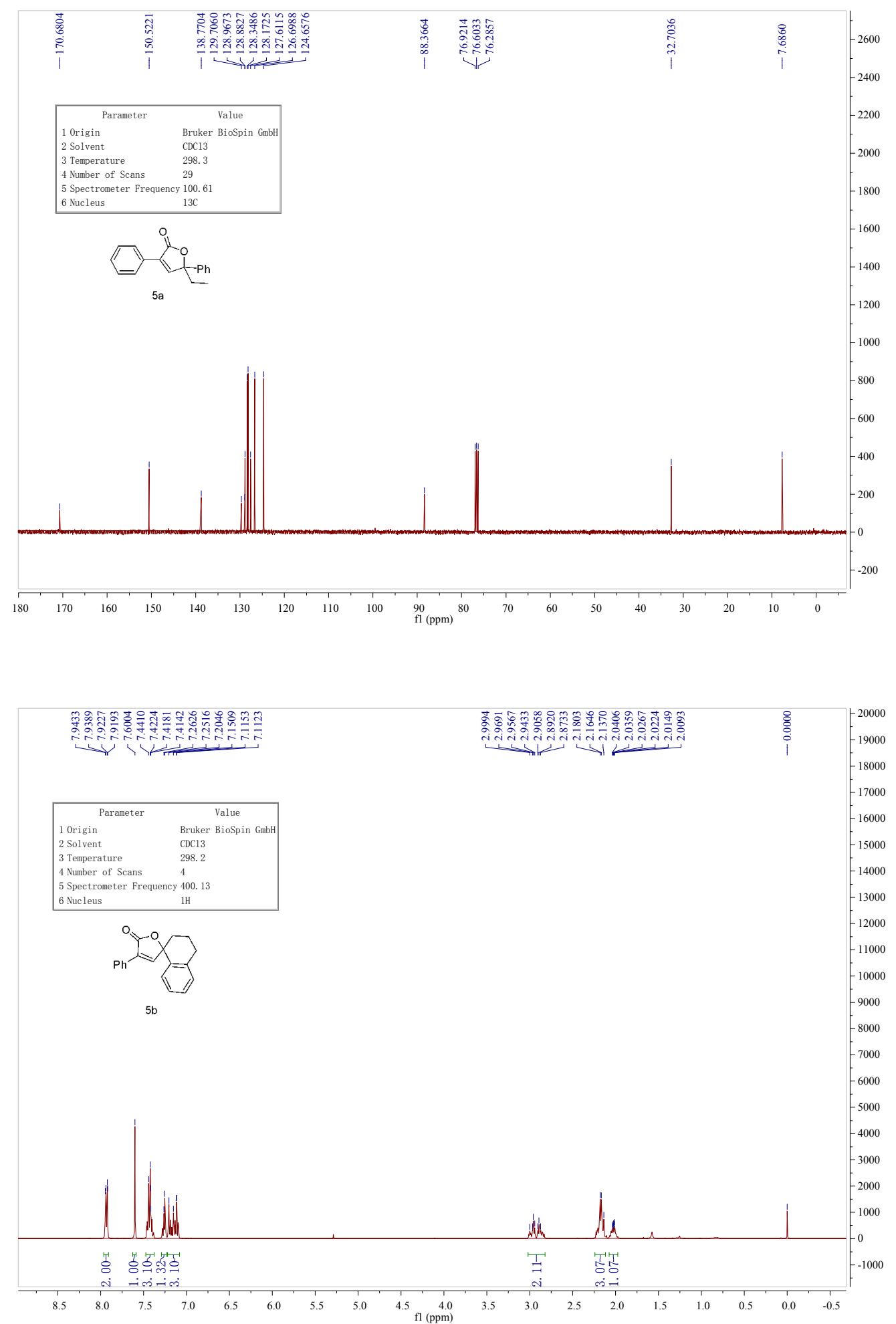

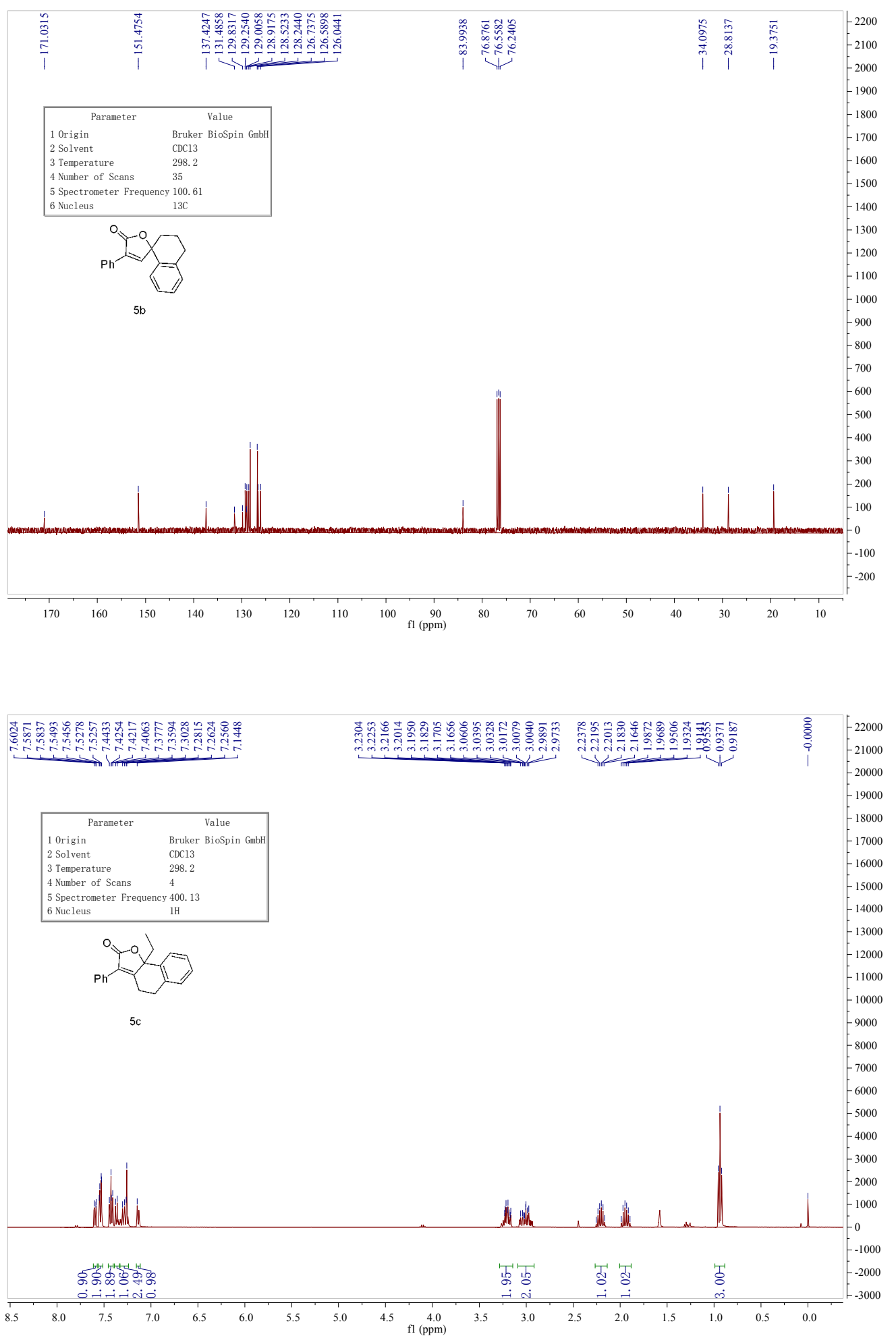

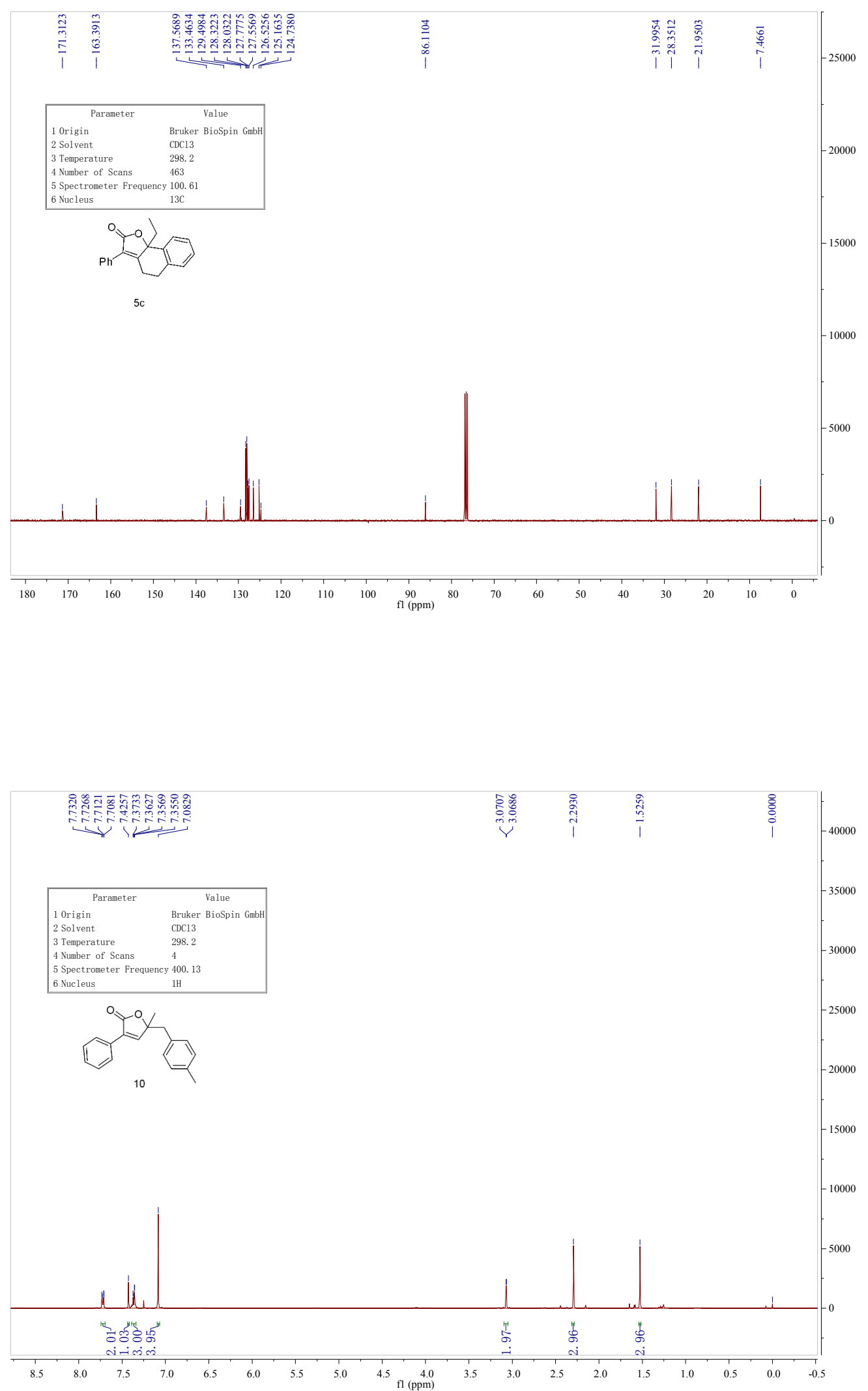


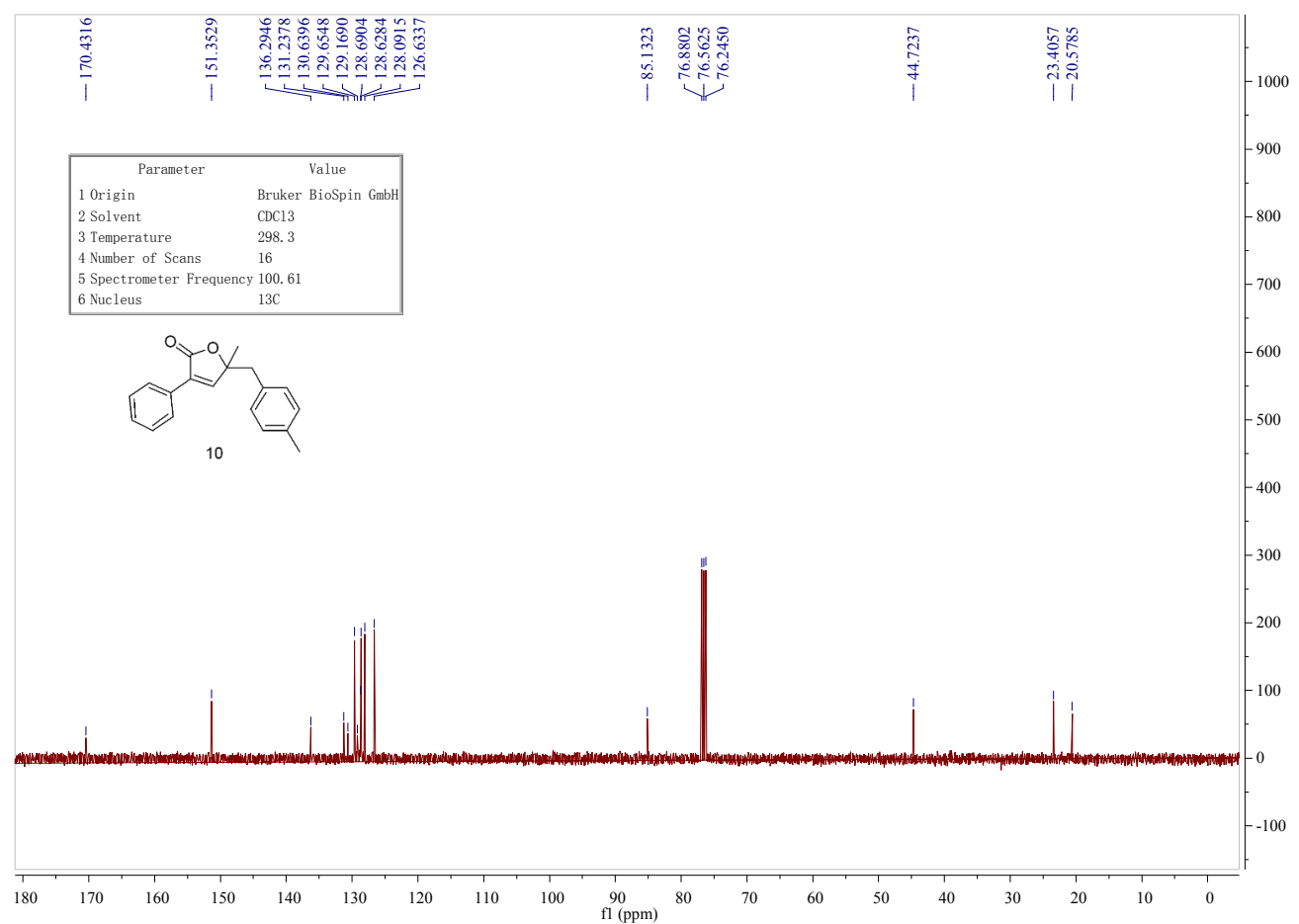

